\title{
Programa de atención a pacientes diabéticos y prediabéticos en farmacia comunitaria: "DayBTS"
}

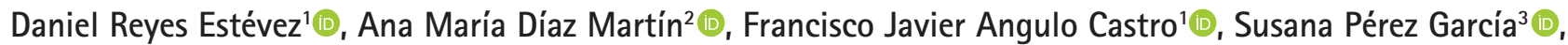 \\ María Victoria Revilla Calvo ${ }^{2}$, Francisco de Asís Miranda Saavedra ${ }^{4}{ }^{(1)}$, Victoria J. Valerón Bergh ${ }^{2} \mathbb{D}_{\text {, }}$ \\ Bartolomé Domínguez del Río Boada ${ }^{5}$ (I)
}

1. Farmacéutico comunitario. 2. Farmacéutica del Colegio Oficial de Farmacéuticos de Santa Cruz de Tenerife (COF SCTFE). 3. Farmacéutica comunitaria. Doctora en Farmacología por la Universidad de Valladolid. 4. Farmacéutico comunitario. Secretario del COF SCTFE. 5. Farmacéutico comunitario. Presidente de la delegación canaria de SEFAC.

\section{PALABRAS CLAVE}

Diabetes mellitus, servicios comunitarios de farmacia, cumplimiento y adherencia al tratamiento

\section{ABREVIATURAS}

AVAC: años de vida ajustados a calidad

AVG: años de vida ganados CRD: cuaderno electrónico de recogida de datos CVRS: calidad de vida relacionada con la salud FID: Federación Internacional de Diabetes

GRD: grupos relacionados con el diagnóstico

HbA1c: hemoglobina glicosilada; ZAP: zona de atención personalizada

IMC: índice de masa corporal OMS: Organización Mundial de la Salud

PRM: perspectiva de uso racional del tratamiento

RGPD: Reglamento General de Protección de Datos

RUM: revisión del uso de la medicación

SEFAC: Sociedad Española de Farmacia Familiar y Comunitaria SFT: seguimiento

farmacoterapéutico

SPFA: servicios profesionales farmacéuticos asistenciales V0,V1,V2,V3,V4,V5,V6: visitas

\section{KEYWORDS}

Diabetes mellitus, pharmacy community services, treatment completion and compliance

\section{RESUMEN}

Justificación: en España hay 6 millones de personas con diabetes. Canarias está 1,86 puntos porcentuales por encima de la media nacional. Aunque haya mejor conocimiento de la diabetes, el número de pacientes sigue aumentando. El farmacéutico comunitario puede jugar un papel importante a través de su formación académica como sanitario experto en el medicamento y por su cercanía al paciente. Por ello, son importantes proyectos de diseño y estandarización de servicios de seguimiento farmacoterapéutico en diabetes más un abordaje multidisciplinar.

Objetivo: diseño de un servicio para atención a pacientes diabéticos y prediabéticos en farmacia comunitaria denominado DayBTS.

Población diana: mayores de edad que acuden a la farmacia y cumplen al menos uno de estos requisitos: tratamiento con medicación antidiabética, presenten sobrepeso u obesidad, hipertensión arterial o hipercolesterolemia, presenten antecedentes familiares de diabetes o directamente soliciten el servicio.

Estructura: serie de visitas divididas en dos fases. Fase inicio, comprende 5 visitas. Fase continuación, 2 visitas para seguimiento y nuevas dudas o problemas. Las actividades a realizar de forma general son: educación diabetológica, medición de variables clínicas (glucemia capilar, índice de masa corporal, hemoglobina glicosilada, patrones en glucemia), variables económicas (visitas a atención primaria, urgencias, número de medicamentos utilizados) y variables humanísticas (adherencia al tratamiento, calidad de vida, conocimientos sobre diabetes).

Coste previsto: el coste por visita se estima en función de: tipo de visita, recursos materiales y tiempo del personal farmacéutico. Entre 5,95€ (paciente prediabético) a 20,90€ (paciente diabético) con precio propuesto de $7,44 €$ y $26,13 €$ para margen $20 \%$. El coste del servicio completo sería de 88,31-111€ (Prediabetes-Diabetes).

Treatment program for diabetic and prediabetic patients in community pharmacy: 'DayBTS'

\section{ABSTRACT}

Reason: in Spain there are 6 million people with diabetes. The Canary Islands are 1.86 percentage points above the national average. Although information on diabetes has improved, the number of patients continues to increase. Community pharmacists can play a significant role thanks to their academic training as healthcare providers specialized in medications and their proximity to patients. For this reason, it is important to have projects for the design and standardization of pharmacotherapeutic monitoring services in diabetes, besides a multidisciplinary approach.

Objective: design of a treatment service for diabetic and prediabetic patients in community pharmacy called DayBTS.

Target population: older people who visit the pharmacy and meet at least one of the following requirements: treatment with antidiabetic medication, are overweight or obese, arterial hypertension or hypercholesterolemia, with family history of diabetes or who ask for the service directly.
Recibido: 10/2/2020

Aceptado: 12/6/2020

Disponible online: 22/7/2020
Financiación: ninguna.

Conflicto de intereses: ninguno.

Cite este artículo como: Reyes D, Diaz AM, Angulo FJ, Pérez S, Revilla MV, Miranda FA, Valerón VJ, Domínguez B. Programa de atención a pacientes diabéticos y prediabéticos en farmacia comunitaria: 'DayBTS.' Farmacéuticos Comunitarios. 2020 Jul 22;12(3):21-50. doi:10.33620/FC.2173-9218.(2020/Nol12).003.04

Correspondencia: Daniel Reyes Estévez (leinad_77@hotmail.com).

ISSN 1885-8619 @SEFAC (Sociedad Española de Farmacia Familiar y Comunitaria). Todos los derechos reservados. 
Structure: series of visits in four phases. Initial phase, includes 5 visits. Continuation phase, 2 visits for monitoring and new doubts or issues. The activities to perform in general are as follows: diabetes education, measurement of clinical variables (blood glucose, body mass index, glycosylated hemoglobin, glycemic patterns), economic variables (visits at primary care, acute care, number of medications used) and human variables (treatment compliance, quality of life, knowledge of diabetes).

Expected cost: the cost per visit is estimated according to: type of visit, material supplies and pharmaceutical staff time. Between $5.95 €$ (prediabetic patient) and $20.90 €$ (diabetic patient) with cost proposed $7.44 €$ and $26.13 €$ for a $20 \%$ margin. The cost of the complete service would be 88.31-111€ (Prediabetes-Diabetes).

\section{Introducción}

La diabetes mellitus (DM) es uno de los mayores problemas de salud mundial del siglo XXI. La Organización Mundial de la Salud (OMS) define esta enfermedad como una afección crónica que aparece cuando el páncreas no produce suficiente insulina o el organismo no consigue utilizarla de manera eficaz (1). La aparición y severidad de complicaciones crónicas viene determinada por el grado de control glucémico y de la presencia asociada de otros factores de riesgo cardiovasculares (2).

A pesar de un mejor conocimiento de la diabetes mellitus (DM) y de nuevos avances tanto en el tratamiento como su prevención, el número de personas afectadas por esta enfermedad sigue aumentando.

En el mundo, según el Informe mundial sobre la diabetes OMS (1), la prevalencia de la diabetes se ha duplicado desde 1980. Atendiendo a los datos autoreferidos aportados en la Encuesta Nacional de Salud 2017 (3) la prevalencia de la diabetes es del 7,8 \%. Según la Encuesta de salud en Canarias 2015 (4), la diabetes se encontraba 1,9 puntos porcentuales por encima de la media de diabetes en nuestro país (España 7,8 \% y en Canarias 9,7 \%). En el mundo, la prevalencia de diabetes se estima en el 9,3\% y la tolerancia anormal a la glucosa, paso previo a la diabetes, es el 7,5\%. Según el Atlas de la diabetes de la FID 2019, se prevé que la prevalencia de la diabetes en Europa sea del 9,8 \% en 2025 (5).

Un porcentaje elevado de diabéticos desconoce que lo son. El estudioDi@betes encontró una prevalencia total del 13,8 y casi la mitad de los pacientes, el $6 \%$, lo desconocía (6).

La diabetes fue la causa declarada de defunción de 9.921 personas en España en 2018 (7). Al mismo tiempo la diabetes es un factor de riesgo muy importante para morir por problemas cardiovasculares. El $50 \%$ de ellos muere por causas cardiovasculares con un riesgo relativo de 2 (8).

La alta prevalencia de DM, junto con su carácter crónico y las numerosas complicaciones asociadas, supone un enorme gasto económico para los sistemas sanitarios y para el propio paciente. En España, en el año 2018, el porcentaje del presupuesto sanitario empleado fue del 9,2 \% (9). El coste sanitario de un paciente diabético es un 59,9\% más alto que el de un paciente no diabético (10).

\section{Justificación}

Por ello, esta enfermedad supone un desafío significativo para los sistemas sanitarios, siendo primordial la prevención, detección y control de los factores de riesgo de la patología (sobrepeso, hipertensión, dislipidemia, etc.) y del fomento de los hábitos de vida saludables.

Es a nivel de atención primaria donde están creadas las condiciones para la detección, el diagnóstico, seguimiento y control adecuados de los pacientes con DM. El farmacéutico comunitario puede jugar un papel importante. Posee una sólida formación académica como profesional sanitario experto en el medicamento. Al mismo tiempo, su cercanía al paciente le facilita investigar el conocimiento de su enfermedad y recomendar hábitos de vida al paciente que impidan el desarrollo de la DM. No olvidemos que es el farmacéutico comunitario el ultimo sanitario de la cadena antes de que el paciente se tome su medicación. España posee 22.071 (diciembre de 2018) farmacias comunitarias a disposición de su población, localizadas en lugares de fácil acceso y repartidas por toda la geografía nacional, con un amplio horario de atención al usuario y sin necesidad de cita previa para consultas al farmacéutico.

El farmacéutico comunitario tiene los conocimientos, herramientas $\mathrm{y}$ condiciones ideales para desempeñar una labor de identificación de enfermos potenciales (detección precoz) y seguimiento de los ya diagnosticados. Además, muchos de los diabéticos son pacientes polimedicados, tratados en ocasiones por especialistas sin relación entre sí y que, por tanto, prescriben sin el conocimiento del tratamiento global recibido por el paciente. En este caso la participación del farmacéutico es especialmente importante en la identificación de posibles problemas relacionados con la farmacoterapia.

Los beneficios obtenidos y la importancia de un seguimiento farmacoterapéutico de pacientes con diabetes por parte de un farmacéutico no es nada nuevo y es cada vez más visible a nivel mundial. Existen proyectos y estudios de coordinación multidisciplinar entre médicos de atención primaria, endocrinos, farmacéuticos comunitarios y pacientes que trabajan de manera conjunta para mejorar el abordaje preventivo y terapéutico de la DM $(11,12)$, así como proyectos educativos dirigidos al paciente con DM (12). Como ejemplo, en Alviobeira (Portugal), Figueiredo et al. El estudio concluye con una mejora del conocimiento de la enfermedad por parte del paciente, la optimización de la terapéutica y la disminución de resultados clínicos negativos de los participantes (13-18).

Es por ello importante el desarrollo de proyectos que incluyan dichos seguimientos y aporten resultados necesarios para apoyar y facilitar la implantación y estandarización de dicho servicio de seguimiento farmacoterapéutico a pacientes con diabetes. 


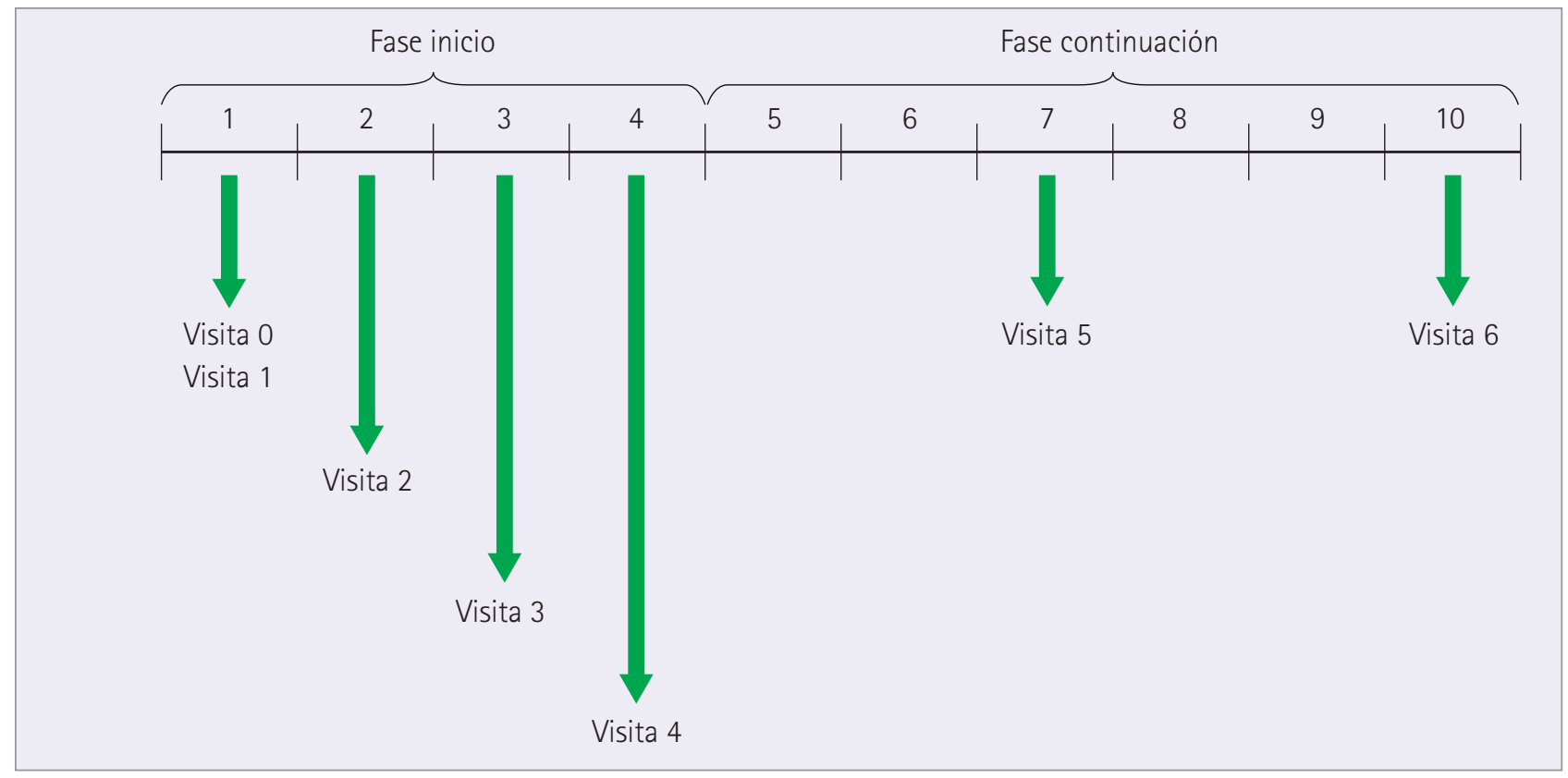

Figura 1 Estructura del programa

\section{Objetivo}

Objetivo principal

- Diseño de un servicio de atención a pacientes diabéticos y prediabéticos en farmacia comunitaria denominado DayBTS.

\section{Objetivos secundarios}

- Detectar posibles casos de diabetes y prediabetes no diagnosticados.

- Hacer un seguimiento de los niveles de hemoglobina glicosilada (HbA1c) y glucemias del paciente.

- Promover el uso racional de los medicamentos.

- Evaluar falta de adherencia al tratamiento farmacológico e intentar corregirla.

- Realizar educación diabetológica: ejercicio físico, educación nutricional, conocimiento de la enfermedad y sus complicaciones.

\section{Aplicabilidad de los resultados}

Según datos del estudio Di@bet.es (28), el porcentaje de pacientes con alteraciones de la glucosa basal en España es del 12,6\% y el porcentaje de diabetes es del 13,8\%. Esto hace un total de $26,4 \%$ de la población española a la que se le podrá aplicar este servicio.

Este servicio se podrá realizar en cualquier tipo de farmacia comunitaria, rural o urbana que cuente con zona de atención personalizada (ZAP) para su desarrollo, formación adecuada y material logístico necesario.
Se beneficiarán de ello los pacientes incluidos en el mismo, así como el resto de profesionales sanitarios implicados en su cuidado.

\section{Diseño}

\section{Material y métodos}

El servicio se basa en una serie de visitas divididas en dos fases. Fase de inicio, que comprende una serie de visitas (V0 a la V4) en las que se van tratando diferentes aspectos. Fase de continuación, con visitas más espaciadas en el tiempo, como seguimiento y para nuevas dudas o problemas derivados de la fase 1 . El procedimiento general puede verse en la figura $1 \mathrm{y}$ el detalle de las visitas en el apartado "desarrollo del servicio".

\section{Procedimientos}

Las actividades que se desarrollan en las diferentes visitas programadas, especificadas en la tabla 1, son:

Educación diabetológica: formación en alimentación, régimen/edulcorantes, hipoglucemia y descompensaciones hiperglucémicas, técnicas de inyección de insulinas, cuidado de los pies y ojos, enfermedad renal y cardiovascular. Documentos obtenidos de la Fundación de la Sociedad Española de Diabetes (FSED), junto con el laboratorio farmacéutico Menarini (28).

Medida de variables clínicas: $\mathrm{HbA1c}$ glucemia capilar, patrones de cambio de la glucemia, índice masa corporal (IMC) y test de Findrisk.
Registro de variables económicas: visitas a atención primaria, ingresos, urgencia, número de medicamentos, coste del servicio RUM, tiempo de entrevistas y calidad de vida relacionada con la salud (CVRS).

Registro de variables humanisticas: calidad de vida, adherencia al tratamiento y cuestionario ECODI.

Logística necesaria para el desarrollo del servicio

Material de divulgación: carteles, folleto descriptivo del servicio, que serán entregados por el patrocinador a las farmacias o bien corresponderá a las farmacias su impresión.

Protocolo: descripción del servicio que incluye de forma detallada su desarrollo y procedimientos de trabajo.

Formación al farmacéutico: los farmacéuticos interesados en participar en el servicio deben contar con la siguiente preparación:

- Introducción e informe del servicio, presentación en diapositivas del servicio, incluyendo formación sobre el cuaderno electrónico de recogida de datos (ver apartado Desarrollo del servicio: cuaderno de recogida de datos electrónicos).

- Formación mediante acceso a un curso (a determinar) acreditado y actualizado en diabetes.

- Manejo de glucómetros y aplicaciones correspondientes y del test de HbA1c.

- Capacitación en Revisión del Uso de los Medicamentos (RUM): programa REVISA de SEFAC o similar. 
Tabla 1 Actividades de visitas programadas

\section{Variable} Definición
Método de medida

MEDIDA DE VARIABLES CLÍNICAS

\begin{tabular}{|c|c|c|}
\hline $\mathrm{HbA1c}$ & $\begin{array}{l}\text { Niveles de glucosa en sangre de los últimos } 3 \text { meses. } \\
\text { La forma predominante de hemoglobina glicada se } \\
\text { conoce como A1c (o HbA1c). Esta prueba se puede } \\
\text { utilizar para detectar una diabetes o riesgo de } \\
\text { desarrollarla. También para monitorizar el tratamiento } \\
\text { de una persona diabética. (18) }\end{array}$ & $\begin{array}{l}\text { Extracción de la muestra de sangre por punción de } \\
\text { una vena del antebrazo o por punción en un dedo } \\
\text { (capilar) con una lanceta. Valores indicio: } \\
\text { - Valor normal HbA1c }<5,7 \% \\
\text { - Valor prediabetes } \mathrm{HbA} 1 \mathrm{c} \text { entre 5,7-6,4\% } \\
\text { - Valor diabetes HbA1c } \geq 6,5 \% \text { Medidor: sistema de } \\
\text { análisis múltiple de HbA1c. (19) }\end{array}$ \\
\hline Glucemia & $\begin{array}{l}\text { Glucemia: nivel de azúcar existente en la sangre. La } \\
\text { glucemia se suele medir en (mg/dl). G. Capilar: prueba } \\
\text { para medir la cantidad de azúcar en la sangre a través } \\
\text { de una pequeña muestra de sangre extraida de los } \\
\text { vasos capilares. (20) }\end{array}$ & $\begin{array}{l}\text { Medidores de glucemia capilar: dispositivos para } \\
\text { analizar los niveles de glucosa en sangre. Medidores } \\
\text { consultados. }\end{array}$ \\
\hline $\begin{array}{l}\text { Patrones de } \\
\text { cambio en la } \\
\text { glucemia }\end{array}$ & $\begin{array}{l}\text { Identificar patrones de hiper/hipoglucemias } \\
\text { relacionados con los hábitos de vida del paciente, } \\
\text { entre visitas. }\end{array}$ & $\begin{array}{l}\text { Aplicación móvil y web que funciona con los } \\
\text { medidores. Distintos modelos consultados de varios } \\
\text { laboratorios. }\end{array}$ \\
\hline IMC & $\begin{array}{l}\text { El índice de masa corporal (IMC). Actualmente es el } \\
\text { método de referencia como parámetro de obesidad } \\
\text { utilizado en todos los estudios clínicos, avalada por la } \\
\text { OMS, por ser el que mejor correlación guarda con el } \\
\text { porcentaje de grasa corporal en el adulto. (21) }\end{array}$ & $\begin{array}{l}\text { Medida de peso en } \mathrm{kg} / \mathrm{el} \text { cuadrado de la talla en } \\
\text { metros }\left(\mathrm{kg} / \mathrm{m}^{2}\right) \text { - La OMS define el sobrepeso como un } \\
\text { IMC igual o superior a } 25 \text {. }\end{array}$ \\
\hline Test de Findrisk & $\begin{array}{l}\text { Regla de predicción clínica a partir de diferentes } \\
\text { poblaciones. El FINnish Diabetes RIsk SCore (FINDRISK) } \\
\text { tiene múltiples ventajas sobre otras escalas de riesgo y } \\
\text { aporta una capacidad razonablemente alta de predecir } \\
\text { la DM no diagnosticada y la prediabetes. (22) }\end{array}$ & $\begin{array}{l}\text { Medida directa al inicio y al final del estudio. El } \\
\text { cuestionario FINDRISK valora } 8 \text { items: edad, IMC, } \\
\text { perimetro cintura, actividad fisica, alimentación, } \\
\text { uso de medicación antihipertensiva, antecedentes } \\
\text { personales cifras elevadas de glucemia e historia } \\
\text { familiar de diabetes. La puntuación del test establece } \\
\text { el nivel de riesgo de padecer diabetes tipo } 2 \text { en } 10 \\
\text { años. }\end{array}$ \\
\hline
\end{tabular}

REGISTRO DE VARIABLES ECONÓMICAS

\section{Visitas a \\ La entrada del paciente en el proceso asistencial}

atención

primaria,

ingresos,

urgencias

(estimados) puede producirse en cualquiera de los niveles de atención sanitaria.

Dado que la información asistencial está dirigida a cuantificar los diferentes tipos de servicios prestados (p.e. número de estancias, visitas, etc.) y, en algunos casos, a identificar la casuistica atendida (p.e. Grupos Relacionados con el Diagnóstico -GRD- en hospitalización), el coste por servicio prestado o por paciente sólo se puede obtener como un coste medio. (23)

\section{Número de} medicamentos, PRM (perspectiva de uso racional del tratamiento)
El uso racional implica que los pacientes reciban los medicamentos adecuados a sus necesidades clínicas, con las dosis precisas según sus características y durante el periodo de tiempo apropiado. Todo ello con el menor coste posible para ellos y para la comunidad. (24)
Pregunta directa al paciente:

Número de visitas a atención primaria: sí o no ( $\left.\begin{array}{lll}1 & 0 & 0\end{array}\right)$.

Número de visitas a urgencias (no ingreso): sí o no $\left(\begin{array}{lll}1 & \text { o } & 0\end{array}\right)$.

Número de ingresos: sí o no ( 1 o 0). 


\begin{tabular}{|c|c|c|}
\hline $\begin{array}{l}\text { Coste del servicio } \\
\text { RUM }\end{array}$ & $\begin{array}{l}\text { Este SPFA tiene un protocolo y procedimientos con un coste } \\
\text { conocido. }\end{array}$ & $\begin{array}{l}\text { Contar el valor del servicio profesional } \\
\text { cada vez que se realiza en los pacientes } \\
\text { diabéticos. }\end{array}$ \\
\hline $\begin{array}{l}\text { Tiempo de } \\
\text { entrevistas }\end{array}$ & Tiempo de duración de la entrevista mensual de los pacientes. & $\begin{array}{l}\text { Medida y registro del tiempo de cada visita, } \\
\text { para estimar coste profesional. }\end{array}$ \\
\hline $\begin{array}{l}\text { Calidad de vida } \\
\text { relacionada con la } \\
\text { salud (CVRS). } \\
\text { Para cálculo de } \\
\text { la utilidad en } \\
\text { su dimensión } \\
\text { económica. Valor } \\
\text { cuantitativo. }\end{array}$ & $\begin{array}{l}\text { El cálculo de la CVRS conlleva la medición de varias } \\
\text { dimensiones, principalmente el funcionamiento fisico, social } \\
\text { y cognitivo, la movilidad y el cuidado personal y el bienestar } \\
\text { emocional. Para el análisis coste-utilidad es adecuado } \\
\text { porque proporciona valores de la utilidad de un estado de } \\
\text { salud. La utilidad es el valor del bienestar percibido por el } \\
\text { paciente para un estado de salud completo. }\end{array}$ & $\begin{array}{l}\text { Cuestionario directo EuroOol-5D } \\
\text { (EQ-5D). Este instrumento presenta } \\
5 \text { preguntas, que analizan otras tantas } \\
\text { dimensiones ofreciendo finalmente un } \\
\text { valor de entre } 0 \text { y } 1 \text { que se corresponde } \\
\text { con la utilidad del estado de salud. } \\
\text { Adicionalmente, existe una escala visual } \\
\text { analógica, con una línea vertical dividida } \\
\text { en cien partes iguales, en donde el nivel } \\
100 \text { se corresponde con el mejor estado de } \\
\text { salud imaginable y el 0, con el peor. } \\
\text { A partir del valor de la utilidad obtenido, } \\
\text { se transforma la supervivencia general } \\
\text {-expresada en años de vida ganados o } \\
\text { AVG- en supervivencia ajustada a calidad } \\
\text {-expresada en años de vida ajustados a } \\
\text { calidad o AVAC. (25) }\end{array}$ \\
\hline
\end{tabular}

REGISTRO DE VARIABLES HUMANÍSTICAS

\begin{tabular}{|c|c|c|}
\hline $\begin{array}{l}\text { Calidad de vida } \\
\text { relacionada con la } \\
\text { salud (CVRS) } \\
\text { Observación de } \\
\text { la utilidad en } \\
\text { su dimensión } \\
\text { humanística valor } \\
\text { cualitativo }\end{array}$ & $\begin{array}{l}\text { El cálculo de la CVRS conlleva la medición de varias } \\
\text { dimensiones, principalmente el funcionamiento físico, social } \\
\text { y cognitivo, la movilidad y el cuidado personal y el bienestar } \\
\text { emocional. La utilidad es el valor del bienestar percibido por } \\
\text { el paciente para un estado de salud completo. (Ver arriba) }\end{array}$ & $\begin{array}{l}\text { Cuestionario directo EuroQol-5D } \\
\text { (EQ-5D). Este instrumento presenta } \\
5 \text { preguntas, que analizan otras tantas } \\
\text { dimensiones ofreciendo finalmente un } \\
\text { valor de entre } 0 \text { y } 1 \text {. Adicionalmente, existe } \\
\text { una escala visual analógica, con una línea } \\
\text { vertical dividida en cien partes iguales, en } \\
\text { donde el nivel } 100 \text { se corresponde con el } \\
\text { mejor estado de salud imaginable y el } 0 \text {, } \\
\text { con el peor. (25) }\end{array}$ \\
\hline $\begin{array}{l}\text { Adherencia al } \\
\text { tratamiento }\end{array}$ & $\begin{array}{l}\text { Adherencia terapéutica como el "servicio profesional } \\
\text { farmacéutico asistencial en el que el farmacéutico, ante } \\
\text { las potenciales dificultades que puede presentar un paciente } \\
\text { en el uso de su terapia, identifica las causas, y mediante } \\
\text { su intervención, en su caso en coordinación con otros } \\
\text { agentes sanitarios, colabora con el paciente para que siga } \\
\text { las recomendaciones sobre el adecuado proceso de uso de } \\
\text { los medicamentos y productos de salud, con los hábitos } \\
\text { higiénico-dietéticos y/o con el estilo de vida, para conseguir } \\
\text { los resultados esperados de su tratamiento". Definición FORO } \\
\text { de Atención Farmacéutica 2019. (27) }\end{array}$ & $\begin{array}{l}\text { Combinación de varios métodos indirectos } \\
\text { para su estimación. } \\
\text { Registrar fechas de dispensación } \\
+ \text { test de Morisky (Adherencia) } \\
+ \text { grado de control de la enfermedad con } \\
\text { la glucemia } \\
\text { + adherencia en RUM, si procede. (26) }\end{array}$ \\
\hline $\begin{array}{l}\text { Cuestionario } \\
\text { ECODI }\end{array}$ & Encuesta sobre Conocimientos de Diabetes. & $\begin{array}{l}\text { Realización del cuestionario, por el } \\
\text { farmacéutico. Obtención del grado de } \\
\text { conocimiento de la enfermedad en varias } \\
\text { visitas, en las que va a ir recibiendo } \\
\text { información y formación. }\end{array}$ \\
\hline
\end{tabular}




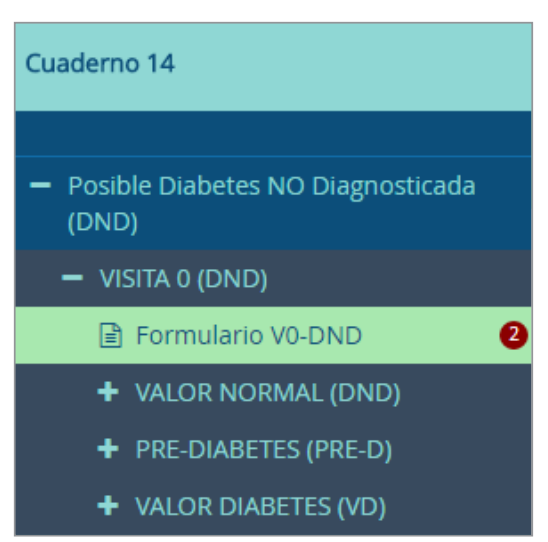

Figura 2 eCRD. Tipo de paciente

\section{Cuaderno de recogida de datos} electrónicos eCRD

Para el desarrollo de las distintas actividades, incluyendo el registro de variables, se utilizará un cuaderno de recogida de datos electrónico (eCRD) (figuras 2 y 3).

Pieza esencial durante el desarrollo de una investigación clínica. Se trata de una herramienta digital que permite seguir el protocolo diseñado. Organizada en apartados que se corresponden con las distintas visitas, además incorpora los diferentes anexos y cuestionarios para que puedan ser descargados y utilizados por el farmacéutico. Es destacable el hecho de que permite guiar al farmacéutico en su proceder y almacenar los valores obtenidos del paciente a lo largo del tiempo, para poder desarrollar un seguimiento adecuado. Del mismo modo, la posibilidad de almacenar los diferentes datos del paciente nos permite su posterior explotación a nivel de investigación.

En cuanto a la protección de datos, todos los introducidos serán cifrados y anónimos. La empresa propietaria del servidor (Inetsys), titular de ShareCRF, que es el software de creación de cuadernos de recogida de datos electrónicos, se compromete a almacenar los datos introducidos por los usuarios registrados y ofrecerlos a ellos. Sin más fin que garantizar la protección de la intimidad personal y el tratamiento confidencial de los datos personales que resulten de la actividad de investigación conforme a lo dispuesto en la Ley Orgánica 3/2018, de 5 de diciembre de Protección de Datos Personales y garantía de los derechos digitales, y a la aplicación de del Reglamento (UE) 2016/679 del Parlamento europeo y del Consejo de 27 de abril de 2016 de Protección de Datos (RGPD).

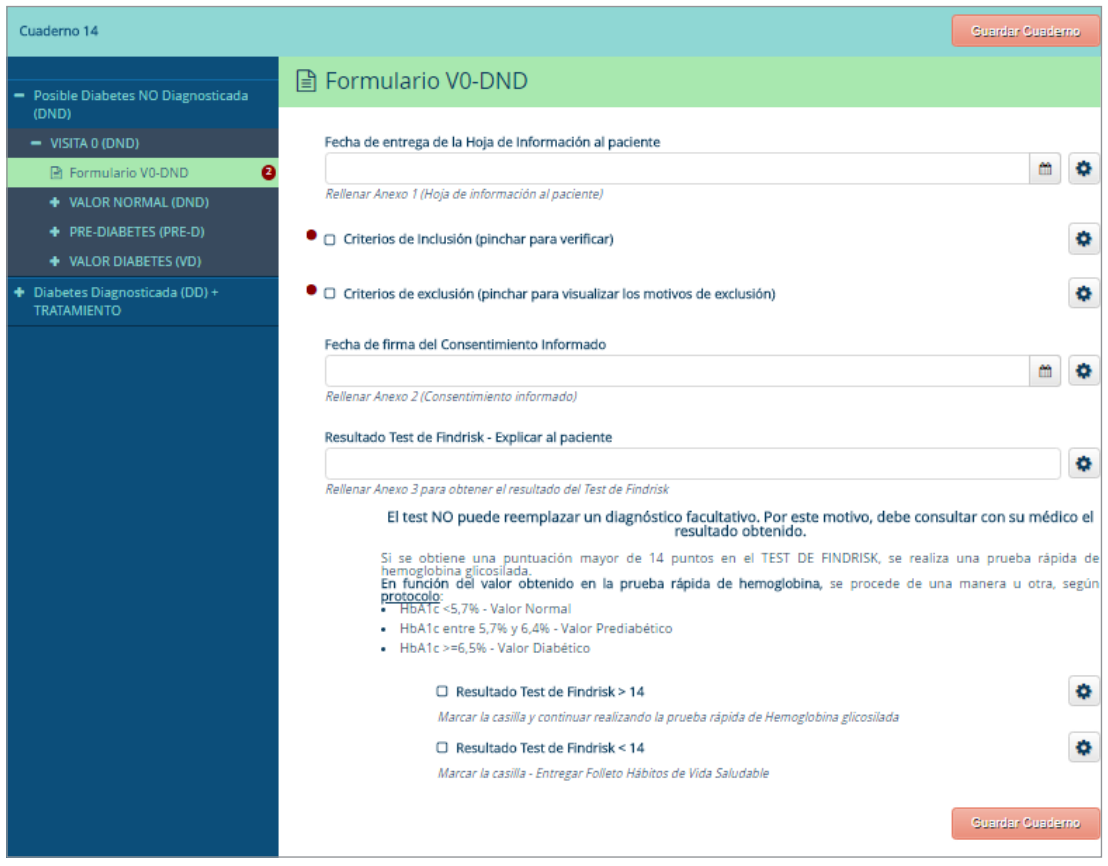

Figura 3 eCRD. Formulario VO

\section{Población diana}

Se ofertará el servicio a todas las personas mayores de edad que cumplan al menos uno de los siguientes requisitos:

- Acudan a la farmacia y lo soliciten.

- Que recojan medicación antidiabética.

- Que presenten sobrepeso u obesidad.
- Que presenten hipertensión arterial o hipercolesterolemia.

- Que presenten antecedentes familiares de diabetes.

\section{Desarrollo del servicio}

A través de la figura 4 , se define la entrada al servicio en base a las características del paciente mediante un algoritmo de decisión.

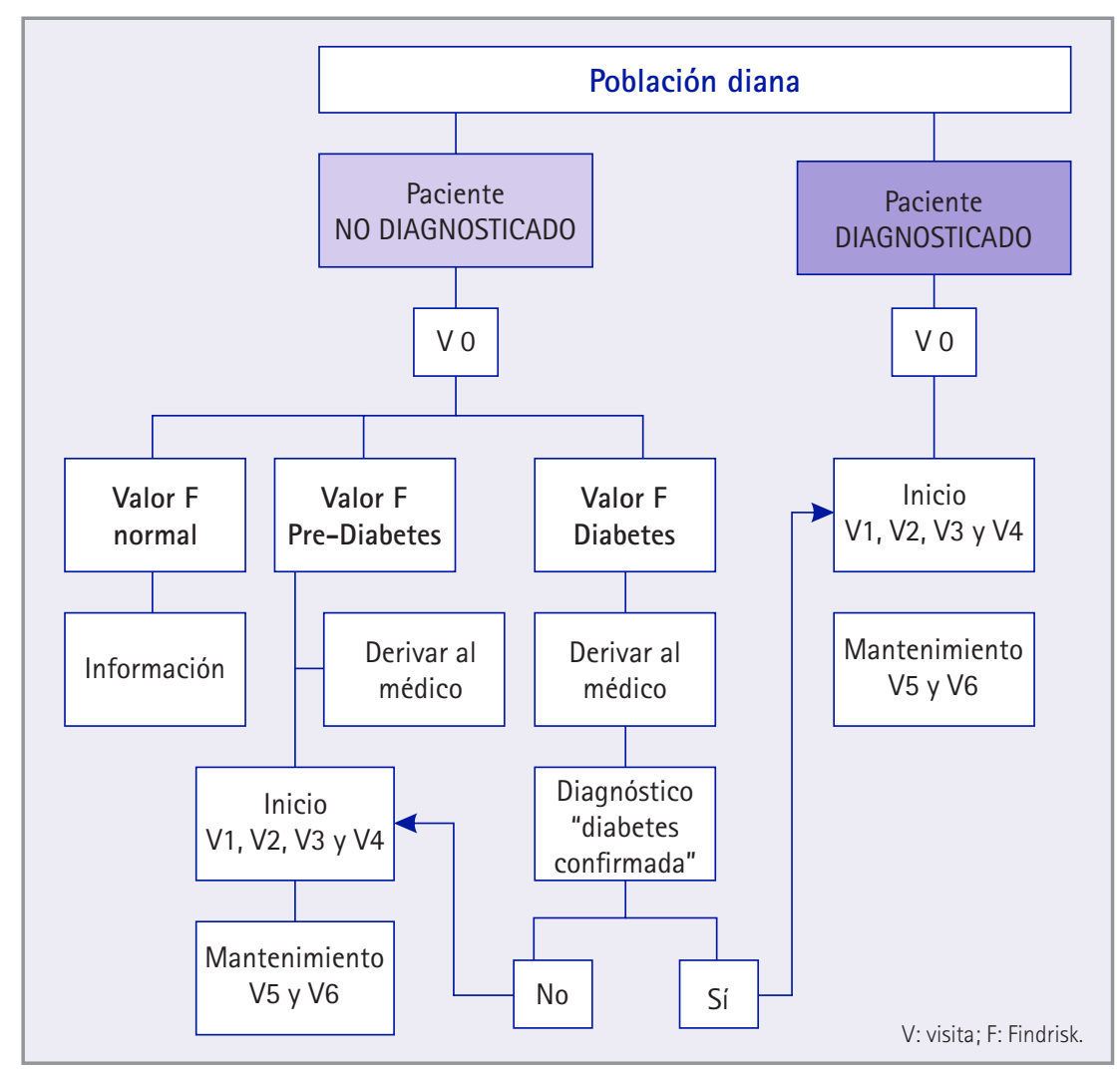

Figura 4. Algoritmo de decisión 


\section{DESCRIPCIÓN DE VISITAS}

1. Población diana

(Pacientes no diagnosticados)

\section{FASE DE INICIO}

Visita 0 (VO)

1. Ofertar el servicio de forma oral y por escrito mediante la hoja de información (anexo 1) a las personas que cumplen con los requisitos de nuestra población diana, pero que no estén diagnosticados de diabetes.

2. Solicitar Consentimiento Informado (anexo 2).

3. Realizar el Test de Findrisk (anexo 3).

- Resultado <15: paciente en riesgo bajo-moderado de padecer diabetes. Se explica al paciente lo que significa la cifra obtenida (anexo 4) y se entrega material informativo sobre alimentación y diabetes (28). El paciente sale del servicio.

- Resultado $\geq 15$ : paciente de alto riesgo de padecer diabetes. Además de explicar al paciente lo que significa la cifra obtenida (anexo 4), se procederá a realizar un test rápido de $\mathrm{HbA} 1 \mathrm{c}$ $\mathrm{y}$ en función del resultado podemos encontrar tres situaciones: pacientes con valor normal de HbA1c (A), pacientes con valor prediabetes (B) y pacientes con valor diabetes (C).

\section{A. Pacientes con valor normal de $\mathrm{HbA} 1 \mathrm{c}(\mathrm{HbA} 1 \mathrm{c}<5,7 \%)$}

4. Se entrega material informativo sobre alimentación y diabetes (28). El paciente sale del servicio.

\section{B. Paciente con valores de}

prediabetes (HbA1c entre 5,7-6,4 \%)

5. Derivar al paciente al médico mediante hoja de derivación (anexo 5).

6. Recoger los datos básicos sobre el paciente incluyendo el IMC (anexo 6).

7. Programar las próximas cuatro visitas (Visita 1 a Visita 4) y continuar con continuación (Visita 5 y Visita 6), mediante calendario del cuaderno electrónico. Cronológicamente la V1 se hará a los 15 días de la V0, y las siguientes visitas se harán mensualmente contando desde la Vo. Las visitas de continuación (V5, V6) se harán cada tres meses.

8. Registrar el tiempo empleado en la Vo (anexo 7). Tiempo estimado de 30 minutos.

\section{Visita 1 (V1)}

1. Registrar si se ha confirmado por el médico el valor de prediabetes.
2. Medir la glucosa capilar. Registrar los valores obtenidos.

3. Rellenar el cuestionario de conocimiento de la enfermedad (anexo 8).

4. Realizar la formación y entregar el material sobre alimentación, diabetes y alimentos de régimen/ edulcorantes (28).

5. Registrar el tiempo empleado en la V1 (anexo 7). Tiempo estimado de 15 minutos.

\section{Visita 2 (V2)}

1. Registrar si se ha confirmado por el médico el valor de prediabetes en caso de no haber obtenido la confirmación en la visita 1 .

2. Medir la glucosa capilar. Registrar el valor obtenido.

3. Realizar la formación y entregar el material sobre hipoglucemia y descompensaciones hiperglucémicas (28-29). Fundamento: prevención y aumentar la percepción de la importancia de seguir las recomendaciones en cuanto a los hábitos de vida saludable.

4. Registrar el tiempo empleado en la V2 (anexo 7). Tiempo estimado de 15 minutos.

\section{Visita 3 (V3)}

1. Registrar si se ha confirmado por el médico el valor de prediabetes en caso no haber obtenido la confirmación en la visita 2 .

2. Medir la glucosa capilar. Registrar el valor obtenido.

3. Realizar la formación y entregar el material sobre cuidado de los pies y de los ojos (28).

4. Registrar el tiempo empleado en la V3 (anexo 7). Tiempo estimado de 15 minutos.

\section{Visita 4 (V4)}

1. Registrar si se ha confirmado por el médico el valor de prediabetes en caso no haber obtenido la confirmación en la visita 3 .

2. Registrar IMC del paciente (incluido en anexo 6).

3. Medir la glucosa capilar y HbA1c. Registrar los valores obtenidos.

4. Rellenar el cuestionario de conocimiento de la enfermedad (anexo 8).

5. Realizar la formación y entregar el material sobre enfermedad renal y enfermedad cardiovascular (28).

6. Registrar el tiempo empleado en la V4 (anexo 7). Tiempo estimado de 15 minutos.

\section{FASE DE CONTINUACIÓN}

\section{Visita 5 (V5)}

1. Medir la glucosa capilar y HbA1c. Registrar los valores obtenidos.

2. Rellenar el cuestionario de conocimiento de la enfermedad. Repasar conceptos y resolver dudas del paciente (anexo 8).

3. Registrar el tiempo empleado en la V5 (anexo 7). Tiempo estimado de 15 minutos.

\section{Visita 6 (V6)}

1. Medir la glucosa capilar y HbA1c. Registrar los valores obtenidos.

2. Rellenar el cuestionario de conocimientos de la enfermedad. Repasar conceptos y resolver dudas del paciente (anexo 8).

3. Registrar IMC del paciente (incluido en anexo 6).

4. Completar la encuesta de satisfacción y retribución (anexo 9). Salvo pregunta 7 del glucómetro.

5. Registrar el tiempo empleado en la V6 (anexo 7). Tiempo estimado de 15 minutos. Finalización del servicio.

\section{Paciente con valores de diabetes (HbA1c $\geq 6,5 \%)$}

6. Derivar al paciente al médico mediante hoja de derivación (anexo 5).

7. Recoger datos básicos sobre el paciente incluyendo el IMC (anexo 6).

8. Registrar tiempo empleado en la Vo (anexo 7). Tiempo estimado de 30 minutos.

9. Esperar al diagnóstico del médico para que el paciente pueda entrar en el servicio como paciente con diabetes, si procede. En este caso, iniciar el registro en V1 paciente diabetes diagnosticada (apartado 2). En caso contrario, iniciar registro en V1 de pacientes con prediabetes (apartado 1B).

10. Información entregada (anexo 4).

\section{Población diana}

(Pacientes diagnosticados)

\section{FASE DE INICIO}

VO

1. Ofertar el servicio de forma oral y por escrito mediante la hoja de información al paciente (anexo 1), a las personas que cumplen con los requisitos de nuestra población diana, que estén diagnosticados de diabetes.

2. Solicitar Consentimiento Informado (anexo 2).

3. Recoger datos básicos sobre el paciente incluyendo el IMC (anexo 6). 
4. Programar las próximas cuatro visitas (Visita 1 a Visita 4) y continuar con mantenimiento (Visita 5 y Visita 6), mediante calendario del cuaderno electrónico.

5. Registrar el tiempo empleado en la Vo (anexo 7). Tiempo estimado de 30 minutos.

\section{V1}

1. Control de visitas a urgencias e ingresos hospitalarios en los 6 meses anteriores a esta visita (anexo 10).

2. Medir la glucosa capilar. Registrar valores obtenidos.

3. Entregar al paciente información para comprobar el manejo del glucómetro y corregir deficiencias.

4. Completar test de conocimiento de la enfermedad (anexo 8).

5. Realizar la formación y entrega de material sobre alimentación, diabetes y alimentos de régimen/edulcorantes y sobre ejercicio físico (28).

6. Completar el test de calidad de vida (anexo 11).

7. Completar el test de adherencia al tratamiento antidiabético: Test Morisky-Green (anexo 12).

8. Registrar el tiempo empleado en la V1 (anexo 7). Tiempo estimado de 20 minutos.

\section{V2}

1. Control de visitas a urgencias e ingresos hospitalarios desde la última visita (anexo 10).

2. Volcar y revisar los datos del glucómetro para verificar hiper/hipoglucemias, intervenir en caso de detección de alteraciones significativas mediante formación o entrega de material, tal como describe el punto 6 , o derivación al médico en caso necesario.

3. Medir la glucosa capilar en caso de no disponer de medidas recientes o en caso de alteraciones significativas al revisar los datos volcados del glucómetro. Registrar valor obtenido.

4. Completar el test de adherencia al tratamiento antidiabético: Test Morisky-Green (anexo 12).

5. Revisión del Uso de los Medicamentos (RUM). Opcional: derivar al seguimiento farmacoterapéutico (SFT) $\mathrm{u}$ otros servicios (anexo 13).

6. Realizar la formación y entrega de material sobre hipoglucemias y descompensaciones hiperglucémicas (28-29). Además, en el caso de pacientes con insulina, entregar y explicar el folleto Técnicas de inyección de insulinas (28).
7. Registrar el tiempo empleado en la V2 (anexo 7). Tiempo estimado de 30 minutos.

\section{V3}

1. Control de las visitas a urgencias e ingresos hospitalarios desde la última visita (anexo 10).

2. Volcar y revisar los datos del glucómetro para verificar hipoglucemias/hiperglucemias, intervenir en caso de detección de alteraciones significativas mediante formación o entrega de material, tal como describe el punto 6, o derivación al médico en caso necesario.

3. Medir la glucosa capilar. Registrar valor obtenido. Medir la glucosa capilar en caso de no disponer de medidas recientes o en caso de alteraciones significativas al revisar los datos volcados del glucómetro. Registrar valor obtenido.

4. Completar el test de adherencia al tratamiento antidiabético: Test Morisky-Green (anexo 12).

5. Realizar la formación y entregar el material sobre cuidado de los pies y de los ojos (28).

6. Registrar el tiempo empleado en la V3 (anexo 7). Tiempo estimado de 20 minutos.

V4

1. Control de visitas a urgencias e ingresos hospitalarios desde la última visita (anexo 10).

2. Registrar IMC del paciente (incluido en anexo 6).

3. Volcar y revisar los datos del glucómetro para verificar hipoglucemias/hiperglucemias, intervenir en caso de detección de alteraciones significativas mediante formación o entrega de material, tal como describe el punto 6 , o derivación al médico en caso necesario.

4. Medir la glucosa capilar y la HbA1c. Registrar valores obtenidos. Medir la glucosa capilar en caso de no disponer de medidas recientes o en caso de alteraciones significativas al revisar los datos volcados del glucómetro. Registrar valor obtenido.

5. Completar el test de adherencia al tratamiento antidiabético: Test Morisky-Green (anexo 12).

6. Revisión del Uso de los Medicamentos (RUM) (anexo 13). Opcional: derivar al SFT u otros servicios.

7. Rellenar el cuestionario de conocimiento de la enfermedad (anexo 8).
8. Realizar la formación y entregar el material sobre enfermedad renal y enfermedad cardiovascular (28).

9. Registrar el tiempo empleado en la V4 (anexo 6). Tiempo estimado de 30 minutos.

10. Cuestionario de satisfacción del glucómetro por parte del farmacéutico (anexo 14), y pregunta 7 del anexo 9.

\section{FASE DE CONTINUACIÓN}

\section{V5}

1. Control de visitas a urgencias e ingresos hospitalarios desde la última visita (anexo 10).

2. Medir la glucosa capilar y la HbA1c. Registrar valores obtenidos.

3. Rellenar el cuestionario de conocimiento de la enfermedad. Repasar conceptos y resolver dudas del paciente (anexo 8).

4. Completar el test de adherencia al tratamiento: Test Morisky-Green (anexo 12).

5. Revisión del Uso de los Medicamentos (RUM). Opcional: derivar al SFT u otros servicios (anexo 13).

6. Registrar el tiempo empleado en V5 (anexo 7). Tiempo estimado de 30 minutos.

\section{V6}

1. Control de visitas a urgencias e ingresos hospitalarios desde la última visita (anexo 10).

2. Medir la glucosa capilar y HbA1c. Registrar los valores obtenidos.

3. Registrar IMC del paciente (incluido en anexo 6).

4. Rellenar el cuestionario de conocimientos de la enfermedad. Repasar conceptos y resolver dudas del paciente (anexo 8).

5. Completar el cuestionario de calidad de vida (anexo 11).

6. Completar el test de adherencia al tratamiento: Test Morisky-Green (anexo 12).

7. Completar la encuesta de satisfacción y retribución (anexo 9 sin la pregunta 7).

8. Revisión del Uso de los Medicamentos (RUM). Opcional: derivar al SFT u otros servicios (anexo 13).

9. Registrar el tiempo empleado en la V6 (anexo 7). Tiempo estimado de 30 minutos. Finalización del servicio. 


\section{Consideraciones éticas}

El riesgo del servicio para el paciente es bajo, ya que, en todo momento, la relación que se mantiene con el mismo es mediante entrevista, cumplimentación de cuestionarios y las decisiones en cuanto al tratamiento (si es necesario) serán tomadas por el médico. La excepción sería la realización de punción capilar para obtener valores de glucemia y hemoglobina glicosilada. Aun así, estas pruebas suponen un riesgo mínimo para el paciente. La finalidad del proyecto es ayudar y apoyar al paciente en su enfermedad a través de formación, revisión de medicación, resolución de dudas etc., por lo que el beneficio para el paciente es alto.

\section{Estimación de costes}

Los costes fijos (CF) son aquellos que no varían con el volumen de producción como el alquiler de local, las amortizaciones y los sueldos. Los costes variables (CV), por el contrario, cambian cuando se registra una variación del volumen de producción, de modo que los costes totales (CT) son:

$$
\mathrm{CT}=\mathrm{CF}+\mathrm{CV}
$$

Los beneficios de una compañía se denominan margen de contribución si no consideramos los costes fijos. En este caso hablamos de coste parcial (CP), que no incluye los costes fijos, en vez de $\mathrm{CT}$.

\section{(Beneficio) $=$ Venta - Coste variable Venta $=$ precio por servicio}

En el corto plazo, en general, los servicios que aportan un margen de contribución positivo (beneficio) no deben eliminarse, ya que, aunque su resultado a coste completo fuese negativo, estarian ayudando a la absorción de costes fijos, que de otra manera recaen sobre otros productos de la farmacia (30).

Por tanto, a la hora de calcular los costes para fijar los precios de un servicio profesional farmacéutico, tendremos en cuenta únicamente los costes variables o aquellos costes que pudiendo ser fijos, sean directamente imputables al servicio en cuestión. (Ej: coste de la mano de obra, siendo un coste fijo para la farmacia, es directamente imputable al servicio).

Estudio de precios, costes y beneficios del servicio en función del

Tabla 2 Resumen coste-precio servicio DayBTS

\begin{tabular}{|l|c|c|c|c|c|}
\hline Visita o servicio & $\begin{array}{c}\text { Tiempo } \\
\text { (min) }\end{array}$ & $\begin{array}{c}\text { Coste } \\
\text { tiempo } \\
\text { (Ct) }\end{array}$ & $\begin{array}{c}\text { Coste } \\
\text { parcial } \\
\text { (CP) }\end{array}$ & $\begin{array}{c}\text { Precio } \\
\text { visita }\end{array}$ & $\begin{array}{c}\text { Beneficio } \\
\text { (20\% } \\
\text { margen)* }\end{array}$ \\
\hline Inicial (pre-diabetes) V0 & 30 & $9,90 €$ & $20,90 €$ & $26,13 €$ & $5,23 €$ \\
\hline Pre-diabetes (V1,V2, V3) & 15 & $4,95 €$ & $5,95 €$ & $7,44 €$ & $1,49 €$ \\
\hline Visita pre-diabetes (V4,V5) & 15 & $4,95 €$ & $15,95 €$ & $19,94 €$ & $3,99 €$ \\
\hline Precio Servicio pre-diabetes (V0 a V5) \\
\hline Inicial (diabetes) V0 & 30 & $9,90 €$ & $10,90 €$ & $13,63 €$ & $2,73 €$ \\
\hline Diabetes sin RUM (V1) & 20 & $6,60 €$ & $17,60 €$ & $22,00 €$ & $4,40 €$ \\
\hline Diabetes con RUM (V2) & 30 & $9,90 €$ & $10,90 €$ & $13,63 €$ & $2,73 €$ \\
\hline Diabetes sin RUM (V3) & 20 & $6,60 €$ & $7,60 €$ & $9,50 €$ & $1,90 €$ \\
\hline Diabetes con RUM (V4,V5) & 30 & $9,90 €$ & $20,90 €$ & $26,13 €$ & $5,23 €$ \\
\hline Precio Servicio diabetes (V0 a V5) & & & $111,00 €$ & $22,20 €$ \\
\hline
\end{tabular}

* Beneficio $=$ Precio visita $-\mathrm{CP}$.

desarrollo de las visitas descritas: estableceremos un coste de la visita en función de la que se trate. Para ello, tendremos que tener en cuenta:

La forma más precisa y que ofrece una mejor visión sobre la salud del negocio es la del margen sobre ventas, de modo que el precio se calcula con la fórmula que detallamos (30):

$$
\text { Precio }=\text { Coste } /(1 \text { - \%margen })
$$

si empleamos el margen de contribución:

$$
\text { Precio = CP / (1 - \% margen })
$$$$
\mathrm{CP} \text { : coste parcial }
$$

\section{Estimación de coste de las visitas}

El sumatorio de los conceptos siguientes, en función del tipo de paciente y visita nos permitirá estimar los costes parciales (CP)

1. Test de hemoglobina glicosilada (10€ media). Unidad/incluido medidor y fungibles.

2. Test de glucemia capilar $(0,5 €$ media). Unidad/incluido medidor y fungibles.

3. Coste salarial del farmacéutico en función del tiempo de la visita (Ct): teniendo en cuenta el número de horas anuales trabajadas, se obtiene que el coste/hora de un farmacéutico adjunto es de $19,63 €(0,33 € /$ minuto).

Se estimó un salario bruto anual de $35.003,5 € / a n ̃ o$, teniendo en cuenta el salario base y el plus de farmacéutico adjunto, además de los seguros sociales (tabla de retribuciones para el año 2016 de la Resolución de 9 de abril de 2014, de la Dirección General de Empleo, por la que se registra y publica el laudo arbitral para oficinas de farmacia, para farmacéutico adjunto. BOE núm. 112 de 8 de mayo de 2014).
4. Otros recursos: papel para impresión de fichas para pacientes, alcohol, algodón $(0,50 €$ por visita $)$.

5. El coste medio asociado a la entrevista RUM (23) es 8,95€ mientras que el coste medio asociado al registro del formulario RUM e informes fue $8,31 €$, resultando un coste medio del tiempo invertido por el farmacéutico en el servicio RUM total de 16,91€. Expresado en términos de mediana, este valor desciende hasta $14,72 €$.

Estimación de precio del servicio para paciente diabético o prediabético en función de los costes calculados

Según datos de los apartados 1 a 4, teniendo en cuenta que el servicio RUM está incluido en el tiempo de la visita, y escogiendo un margen de beneficio del $20 \%$ :

\section{Coste parcial (CP) $=\Sigma$ apartados 1-4 en función de la visita \\ Precio visita $=\mathrm{CP} /$ (1-\%margen)}

\section{Funcionalidad}

Se ha realizado una prueba de funcionalidad para ver si el procedimiento es factible. Durante una semana se ha aplicado en una farmacia a 6 pacientes. La herramienta electrónica fue accesible y el tiempo necesario para las visitas osciló entre 15 y 30 minutos. Los pacientes se mostraron satisfechos con el servicio.

Para refrendar esta prueba, está previsto realizar un estudio piloto en el año 2021 de 10 meses de duración con la participación de 100 farmacias instruidas en todo el territorio nacional. Cada farmacia deberá reclutar un total 10 pacientes, de los cuales 5 serán prediabéticos y 5 diabéticos. Para una muestra representativa de 1.000 pacientes aproximadamente. 


\section{Referencias bibliográficas}

1. WHO. Informe mundial sobre la Diabetes. Última consulta: octubre 2019. https://apps.who.int/iris/bitstream/ handle/10665/204877/WHO_NMH_ NVI_16.3_spa.pdf;jsessionid=1A1F4FC4A2F8C03ED69B7571114E83B2? sequence $=1$.

2. Guía Farmacoterapéutica de la Diabetes Mellitus. Plan Estratégico para el Desarrollo de la ATENCIÓN FARMACÉUTICA'10. Consejo General de Colegio Oficiales de farmacéuticos. Última consulta: octubre 2019. https://botplusweb.portalfarma.com/ documentos/2010/8/23/44205.pdf

3. Instituto Nacional de Estadística (INE). Encuesta Nacional de Salud, 2017. Última consulta: septiembre 2019. https://www.ine.es/dyngs/INEbase/ es/operacion.htm $? \mathrm{c}=$ Estadistica CEtcid $=1254736176783 \mathrm{Ctmenu}=\mathrm{re}-$ sultadosetsecc $=1254736195650$ cti$\mathrm{dp}=1254735573175$

4. Encuesta de Salud de Canarias (ESC2015) http://www.datosdelanzarote.com/ Uploads/d oc/Encuesta-de-Salud-de-Canarias-(2015)-201612051115 4059ESC-2015-Resultados.pdf

5. Diabetes Atlas de la Federación Internacional de Diabetes. Novena edición, 2019. https://diabetesatlas.org/en/resources/

6. Soriguer F, Goday A, Bosch-Comas A, et al. Prevalence of diabetes mellitus and impaired glucose regulation in Spain: the Di@bet.es Study. Diabetologia 55:88-93, 2012. doi:10.1007/ s00125-011-2336-9

7. Instituto Nacional de Estadística. Defunciones según la causa de la muerte. https://www.ine.es/jaxiT3/Datos.htm? $\mathrm{t}=7947$ \#!tabs-tabla

8. Domingo Orozco-Beltrán, Eva Sánchez, Alejandro Garrido, José Antonio Quesada, M. Concepción Carratalá-Munuera, Vicente F. Gil-Guillén. Evolución de la mortalidad por diabetes mellitus en España: análisis del periodo 1998-2013. Rev Esp Cardiol. 2017;70(6):433-443. doi:10.1016/j.recesp.2016.07.007

9. Del Llano Señarís, Juan E. Informe Sanidad Española en cifras 2018. pág 245. http://fundaciongasparcasal.org/publicaciones/Sanidad-espanola-en-cifras-2018.pdf

10. Manel Mata Cases. Coste actual de la diabetes mellitus en España: el estudio eCostesDM2. Mesa redonda: los costes de la DM2. http://www.diabetespractica.com/files/docs/publicaciones/138512825809_Mata.pdf

11. Médicos, farmacéuticos y pacientes se unen para mejorar el abordaje preventivo y terapéutico de la diabetes. Nota de prensa SEFAC 31/10/17. Última consulta: octubre 2018. https:// www.sefac.org/notas-de-prensa/cam- pana-conjunta-para-mejorar-el-abordaje-preventivo-y-terapeutico-de-la-diabetes

12. Eik Filho W, Pastorelli Bonjorno L, Mendes Franco AJ, et al. Evaluation, intervention, and follow-up of patients with diabetes in a primary health care setting in Brazil: the importance of a specialized mobile consultancy. Diabetology \& Metabolic Syndrome. 2016; 8: 56. doi:10.1186/ s13098-016-0173-1

13. Hughes JD, Wibowo Y, Sunderland B, Hoti $\mathrm{K}$. The role of the pharmacist in the management of type 2 diabetes: current insights and future directions. Integrated Pharmacy Research and Practice. 6: 15-27, 2017. doi:10.2147/ IPRP.S103783

14. Figueiredo IV, Móteiro C, Castel-Branco MM, et al. Seguimiento de pacientes con diabetes tipo 2 en una unidad de cuidados primarios de salud. Pharmaceutical Care España 17(2): 3948, 2014. https://www.pharmcareesp. com/index.php/PharmaCARE/article/ view/161

15. Marcelino-Rodríguez I, Elosua R, Rodríguez Pérez MC, et al. On the problem of type 2 diabetes-related mortality in the Canary Islands, Spain. The DARIOS Study. Diabetes Research and Clinical Practice 111:74-82, 2016. doi:10.1016/j.diabres.2015.10.024

16. Estilo de vida y adherencia al tratamiento de la población canaria con diabetes mellitus tipo 2. Cabrera de León A, del Castillo Rodríguez JC, Dominguez Coello S, et al. Revista Española de Salud Pública 83: 567-575, 2009. doi:10.1590/S113557272009000400008

17. Disproportionately high incidence of diabetes-related end-stage renal disease in the Canary Islands. An analysis based on estimated population at risk. Lorenzo V, Boronat M, Saavedra P, et al. Nephrology Dialysis Transplantation 25: 2283-2288, 2010. doi:10.1093/ndt/gfp761

18. Impared fasting glucose. Impaired fasting glucose, ancestry and waistto-height ratio: main predictors of incident diagnosed diabetes in the Canary Islands. Cabrera de León A, Dominguez Coello S, Almeida González D. DiabeticMedicine 29: 399403, 2012. doi:10.1111/j.1464-5491. 2011.03420.x

19. Cortés, M (SEQC-ML). Hemoglobina glicada. 04.12.2017. https://labtestsonline.es/tests/hemoglobina-glicada

20. Suárez Alemán, C; Navarro Moya, F.J; Espinar Toledo, M; Sepúlveda Muñoz, J; Blanco Morgado, J. Guía de uso adecuado de productos para autoanálisis de glucemia capilar. https://bit. ly/2TRG5UK.12/2014
21. Calculadora IMC, peso deseable y cálculo de las necesidades calóricas. Fisterra. https://bit.ly/2BxHOaH. 11/12/2013

22. Serrano Martín, R. ¿Son las reglas predictivas de riesgo la estrategia más adecuada para la detección de pacientes con alto riesgo de diabetes? https://www.redgdps.org/gestor/ upload/GUIA2016/P4.pdf

23. Gómez Bermúdez E, García-Agua Soler N, Jódar Sánchez F, Baixauli Fernández VJ Proyecto 'Revisa ${ }^{\circledR}$ '. Impacto del servicio de revisión del uso de los medicamentos en España. Informe de tiempos y costes. Farmacéuticos Comunitarios. 2017 Sep 30; 9(3):20-24. doi:10.5672/ FC.2173-9218.(2017/Vol9).003.05

24. Baixauli Fernández, VJ. Servicio de revisión del uso de los medicamentos en la farmacia comunitaria. Sociedad Española de Farmacia Familiar y Comunitaria (SEFAC).11/2015. ISBN: 978-84-608-4247-7.

25. Herdmana. M, Badia. X, Berraa. S .El EuroQol-5D: una alternativa sencilla para la medición de la calidad de vida relacionada con la salud en atención primaria. Vol. 28. Núm. 6. Pg. 365446. 11/2001. doi:10.1016/S0212-6567 (01)70406-4

26. Ibarra Barrueta. 0, Morillo Verdugo. R. Lo que debes saber sobre la ADHERENCIA al tratamiento. https://www. sefh.es/bibliotecavirtual/Adherencia2017/libro_ADHERENCIA.pdf

27. Foro de Atención Farmacéutica-Farmacia Comunitaria (Foro AF-FC). Guía práctica para los Servicios Profesionales Farmacéuticos Asistenciales en la Farmacia Comunitaria. Madrid: Consejo General de Colegios Oficiales de Farmacéuticos; 2019.

28. Fundación Sociedad Española de Diabetes (FSED) y Grupo Menarini. Folletos. https://fundacion.sediabetes.org/ index.php/folletos/

29. Servicio canario de la salud. Hojas informativas y recomendaciones para pacientes. Gobierno de Canarias. https://www3.gobiernodecanarias.org/ sanidad/scs/content/c4c91316-a70211e0-a6f1-d5c39b 10ecae/ 19 Hiperglucemia.pdf

30. Delgado Tenorio M. Cómo calcular el precio de venta dado el coste y el margen deseado. 09/2013. https:// manueldelgado.com/como-calcular-el-precio-de-venta-coste-margen/

31. Soriguer F, Valdés S, Rojo G. Sociedad Española de Diabetes. El estudio Di@ bet.es, ¿y ahora qué? doi:10.1016/j. avdiab.2012.06.001

32. Arbaiza Fermini L. Decisiones empresariales a partir del análisis del margen de contribución.05/2017. https:// www.esan.edu.pe/apuntes-empresariales/2017/05/decisiones-empresariales-a-partir-del-analisis-del-margen-de-contribucion/ 
ANEXO 1. Hoja de información al paciente

Nos dirigimos a $\mathrm{Vd}$. para informarle sobre el proyecto de investigación DayBTS: SERVICIO PROFESIONAL PARA EL CONTROL DE LA DIABETES DESDE LA FARMACIA COMUNITARIA

$\mathrm{D} / \mathrm{D}^{\mathrm{a}}$

en su calidad de

de la farmacia

situada en

El objetivo y finalidad del proyecto es evaluar el impacto al implantar un servicio protocolizado de Atención al Paciente diabético o prediabético, desde la farmacia comunitaria, con acciones concretas en cada intervención, mediante el uso de una herramienta informática.

En dicho PROYECTO podrán participar voluntariamente aquellos ciudadanos que así lo estimen y que se comprometan a:

- Entrevistarse al menos una vez al mes con el farmacéutico investigador (previa cita).

- Llevar en la primera cita todos los medicamentos que utiliza y contestar a algunas preguntas sobre ellos y sobre su estado de salud.

Durante la realización del PROYECTO, se le solicitará o aconsejará:

- Responder a las preguntas que el farmacéutico le plantee en citas sucesivas sobre cambios en el estado de salud y medicamentos.

- Facilitar sus datos personales como nombre, dos apellidos, sexo, fecha de nacimiento, DNI, nivel de estudios, situación laboral, tratamientos farmacológicos, problemas de salud, medida de la glucemia el test rápido de hemoglobina glicosilada, presión arterial, visitas al centro de salud, urgencias o ingresos hospitalarios.

En concreto, en caso de hospitalización anterior a su participación en el PROYECTO o durante la participación del mismo, facilitar el coste por GRD (Grupos Relacionados con el Diagnóstico) correspondiente o, en caso de no disponer del mismo, autorizar al hospital para facilitar la citada información al GRUPO INVESTIGADOR otorgando a tal efecto la correspondiente autorización.

- Derivación a otro servicio como Evaluación de Riesgo Cardiovascular, Servicio de Sistema Personal de Dosificación, servicio de Seguimiento Farmacoterapéutico, Deshabituación tabáquica, etc., como resultado de esta primera cita o siguientes.

Su participación en el PROYECTO le permitirá mejorar la información sobre la diabetes, sobre todos los medicamentos que utiliza, aclarar algunas dudas que se le puedan plantear.

Su participación en el PROYECTO no presenta riesgos para Vd.

Para participar en el PROYECTO se le informa de forma inequívoca y precisa de que es imprescindible que $\mathrm{Vd}$. facilite al GRUPO INVESTIGADOR, como único destinatario de los mismos, los datos de carácter personal que se le recaban a través del formulario de consentimiento informado. Si Vd. no facilita dichos datos no podrá participar en el PROYECTO.

El tratamiento, la comunicación y la cesión de los datos de carácter personal de todos los sujetos participantes se ajustará a lo dispuesto en la Ley Orgánica 3/2018, de 5 de diciembre de Protección de Datos Personales y garantía de los derechos digitales, y a la aplicación de del Reglamento (UE) 2016/679 del Parlamento europeo y del Consejo de 27 de abril de 2016 de Protección de Datos (RGPD), por lo que es importante que conozca la siguiente información: 
- Además de los derechos que ya conoce (acceso, modificación, oposición y cancelación de datos) ahora también puede limitar el tratamiento de datos que sean incorrectos, solicitar una copia o que se trasladen a un tercero (portabilidad) los datos que usted. ha facilitado para el estudio. Para ejercitar sus derechos, dirijase al investigador principal del estudio. Le recordamos que los datos no se pueden eliminar, aunque deje de participar en el estudio para garantizar la validez de la investigación y cumplir con los deberes legales y los requisitos de autorización de medicamentos. Así mismo, tiene derecho a dirigirse a la Agencia de Protección de Datos si no quedara satisfecho.

- Tanto el centro como el Promotor y el Investigador son responsables respectivamente del tratamiento de sus datos y se comprometen a cumplir con la normativa de protección de datos en vigor. Los datos recogidos para el estudio estarán identificados mediante un código, de manera que no se incluya información que pueda identificarle, y sólo su médico del estudio/colaboradores podrá relacionar dichos datos con usted y con su historia clínica. Por lo tanto, su identidad no será revelada a ninguna otra persona salvo a las autoridades sanitarias, cuando así lo requieran o en casos de urgencia médica. Los Comités de Ética de la Investigación, los representantes de la Autoridad Sanitaria en materia de inspección y el personal autorizado por el promotor, únicamente podrán acceder para comprobar los datos personales, los procedimientos del estudio clínico y el cumplimiento de las normas de buena práctica clínica (siempre manteniendo la confidencialidad de la información).

El Investigador y el Promotor están obligados a conservar los datos recogidos para el estudio al menos hasta 25 años tras su finalización. Posteriormente, su información personal solo se conservará por el centro para el cuidado de su salud y por el promotor para otros fines de investigación científica si usted hubiera otorgado su consentimiento para ello, y si asi lo permite la ley y requisitos éticos aplicables.

Si Vd. quiere participar en el PROYECTO se le informa que debe consentir expresamente la inclusión de sus datos personales de: nombre, dos apellidos, sexo, fecha de nacimiento, DNI, nivel de estudios, situación laboral, tratamientos farmacológicos, problemas de salud, medida de la glucemia o hemoglobina glicosilada, presión arterial, valoración del estado de la diabetes en su caso, visitas al centro de salud, urgencias o ingresos hospitalarios, asi como los resultantes de la participación en el PROYECTO, en un fichero de datos de carácter personal de la responsabilidad del GRUPO INVESTIGADOR. El destinatario de sus datos personales es exclusivamente el GRUPO INVESTIGADOR. Los farmacéuticos de las farmacias participantes en el PROYECTO, como investigadores del mismo y encargados del GRUPO INVESTIGADOR en el tratamiento de sus datos, accederán e incorporarán en un sistema de registro seguro con nivel alto de seguridad los datos que le son recabados y mantendrán la plena confidencialidad de los mismos de acuerdo con la legislación vigente. El acceso a sus datos de carácter personal queda restringido al MISMO y a los investigadores del PROYECTO como encargados del tratamiento de los mismos, pero siempre manteniendo la confidencialidad de acuerdo a la legislación vigente. Vd. puede ejercitar sus derechos de acceso, rectificación, cancelación, oposición, limitación del tratamiento y portabilidad de sus datos, dirigiéndose personalmente por escrito a la farmacia . o al GRUPO INVESTIGADOR acompañando fotocopia de su DNI o documento acreditativo de su personalidad, a la siguiente dirección de e-mail o por correo ordinario a 
ANEXO 2. Modelo de consentimiento informado del paciente

Farmacia

D/Dña con DN

autorizo al farmacéutico

a prestarme el servicio de control de la diabetes desde la farmacia comunitaria - DayBTS.

Para ello doy mi permiso para registrar los datos personales y sanitarios, de los que no se hará otro uso sin mi consentimiento expreso.

Manifiesto haber sido informado de todo el proceso del servicio, asi como de su coste, y que el servicio se inicia y se lleva a cabo con mi consentimiento.

Por su parte, el farmacéutico D/Dña.

con DNI se compromete a:

- Mantener la privacidad de los datos personales y farmacológicos.

- Realizar este servicio siguiendo las normas establecidas en el Procedimiento Normalizado de Trabajo correspondiente.

- Proporcionar la información necesaria para gestionar de forma adecuada la enfermedad.

Fecha: a de de

De acuerdo con lo establecido en la Ley Orgánica 3/2018, de 5 de diciembre de Protección de Datos Personales y garantía de los derechos digitales, y a la aplicación de del Reglamento (UE) 2016/679 del Parlamento europeo y del Consejo de 27 de abril de 2016 de Protección de Datos (RGPD), los datos que se faciliten se incorporarán al fichero del cual es titular el farmacéutico titular y el GRUPO INVESTIGADOR con la única finalidad de ofrecer una mejor asistencia sanitaria y atención farmacéutica y sobre el cual puede ejercitar sus derechos de acceso, rectificación, cancelación y oposición. 
1. Indica tu edad (*)

1. 0 puntos: Menos de 45 años

2. 2 puntos: Entre $45-54$ años

3. 3 puntos: Entre 55-64 años

4. 4 puntos: Más de 64 años

2. Índice de masa corporal (IMC)

1. 0 puntos: Menos de $25 \mathrm{Kg} / \mathrm{metros}$ cuadrados

2. 1 punto: Entre $25-30 \mathrm{Kg} /$ metros cuadrados

3. 3 puntos: Más de $30 \mathrm{Kg} /$ metros cuadrados

3a. Si Usted es hombre - Perímetro de la cintura medido debajo de las costillas (normalmente a la altura del ombligo)

1. 0 puntos: Menos de $94 \mathrm{~cm}$

2. 3 puntos: Entre $94-102 \mathrm{~cm}$

3. 4 puntos: Más de $102 \mathrm{~cm}$

3b. Si Usted es mujer - Perímetro de la cintura medido debajo de las costillas (normalmente a la altura del ombligo)

1. 0 puntos: Menos de $80 \mathrm{~cm}$

2. 3 puntos: Entre $80-88 \mathrm{~cm}$

3. 4 puntos: Más de $88 \mathrm{~cm}$

4. Normalmente, ¿practica Usted 30 minutos cada día de actividad física en el trabajo y/o en su tiempo libre (incluya la actividad diaria normal)?

1. 0 puntos: Sí

2. 2 puntos: No

5. ¿Con cuánta frecuencia como Usted vegetales o frutas?

1. 0 puntos: Todos los días

2. 1 punto: No todos los dias

6. ¿Ha tomado Usted medicación para la hipertensión con regularidad?

1. O puntos: No

2. 2 puntos: $\mathrm{Si}$

7. ¿Le han encontrado alguna vez niveles altos de glucosa en sangre, por ejemplo, en un examen médico, durante una enfermedad, durante el embarazo?

1. 0 puntos: No

2. 5 puntos: $\mathrm{Sí}$

8. ¿A algún miembro de su familia le han diagnosticado diabetes (tipo 1 ol 2)?

1. O puntos: No

2. 3 puntos: Sí: abuelos, tíos o primos hermanos (pero no: padres, hermanos o hijos)

3. 5 puntos: Si: padres, hermanos o hijos propios

Escala de riesgo total. Sume los puntos de las respuestas contestadas.

El riesgo de desarrollar diabetes tipo 2 durante los próximos 10 años es:

1. Menos de 7 puntos: BAJO - Se estima que 1 de cada 100 pacientes desarrollara la enfermedad.

2. 7-11 puntos: LIGERAMENTE ELEVADO - Se estima que 1 de cada 25 pacientes desarrollará la enfermedad.

3. 12-14 puntos: MODERADO - Se estima que 1 de cada 6 pacientes desarrollará la enfermedad.

4. 15-20 puntos: ALTO - Se estima que 1 de cada 3 pacientes desarrollará la enfermedad.

5. Más de 20 puntos: MUY ALTO - Se estima que 1 de cada 2 pacientes desarrollará la enfermedad.

\section{Observaciones:}

El test FINDRISK no puede reemplazar un diagnóstico facultativo. Por este motivo, debería consultar con su médico el resultado obtenido.

A los pacientes que tengan una puntuación del Test mayor o igual de 15 puntos, es decir riesgo alto o muy alto de padecer diabetes, se les realizará una prueba rápida de hemoglobina glicosilada y en función del valor obtenido en dicha prueba, se procederá de una manera u otra, según protocolo. 


\section{Findrisk (nivel de riesgo de contraer diabetes en los próximos 10 años*)}

\section{Menos de 7 puntos (1 por ciento*)}

Su nivel de riesgo es muy bajo. En su caso no es necesario un cuidado especial o de prevención. Sin embargo, no estaría mal cuidar de su alimentación y realizar suficiente ejercicio.

\section{De 7 a 11 puntos ( 4 por ciento*)}

Para usted es recomendable un poco de cuidado, aunque el nivel de riesgo de contraer una diabetes no es muy alto. Si quiere ir sobre seguro, siga las siguientes reglas:

- En el caso de sobrepeso deberá intentar disminuir su peso en un 7 por ciento.

- Manténgase en actividad, por lo menos, por media hora durante cinco días a la semana.

- La grasa debería constituir como máximo, sólo un 30 por ciento de su alimentación.

- La parte de ácidos grasos no saturados (sobre todo en la grasa animal) no debería sobrepasar del 10 por ciento en su alimentación.

- Consuma diariamente, por lo menos, 30 gramos de fibras vegetales (como las contenidas en productos integrales, verduras y frutas).

\section{De 12 a 14 puntos (17 por ciento*)}

Si usted se encuentra en este grupo de riesgo, no debería postergar, por ningún motivo, el tomar medidas preventivas. En este caso lo pueden ayudar consejos e instrucciones de expertos para cambiar su estilo de vida, los cuales puede aplicarlos usted mismo. Recurra a ayuda profesional si nota que de esa manera no se puede ayudar.

\section{De 15 a 20 puntos (33 por ciento*)}

Su nivel de riesgo es muy alto: una tercera parte de los pacientes que corresponden a este grupo de riesgo contraen diabetes en los próximos 10 años. El subestimar esta situación puede traer graves consecuencias. Lo mejor sería recurrir a ayuda profesional. Haga una prueba de glucemia (azúcar en la sangre) en una farmacia y vaya a hacerse exámenes médicos (checkup a partir de los 35).

\section{Más de 20 puntos (50 por ciento*)}

Existe la necesidad de actuar inmediatamente, ya que es muy posible que usted ya sufra de diabetes. Eso pasa con el 35 por ciento de las personas que se encuentran sobre los 20 puntos. Una simple prueba de glucemia en su farmacia más cercana, por ejemplo, puede servir de ayuda como una información adicional. De todas formas, ésta no reemplaza un diagnóstico del laboratorio para descartar una diabetes ya existente. Por esta razón debería solicitar una consulta médica, inmediatamente.

* El riesgo en porcentaje = 4\% significa, por ejemplo, que 4 de cien personas con este puntaje pueden contraer, en los próximos 10 años, una Diabetes Mellitus Tipo 2. 


\section{Usted puede disminuir el riesgo de la siguiente forma Incluso pequeños cambios en su estilo de vida pueden apoyar su salud}

\section{COMER Y BEBER DE FORMA SALUDABLE}

\section{Más fruta y verdura}

Todos sus alimentos deben contener, en lo posible, mucha fruta y verdura. Lo ideal es que aplaque su hambre solamente con estos alimentos.

\section{Alimentos pobres en grasas}

Al comprar productos lácteos elija las variantes con poca grasa. Coma carnes y embutidos magros, pero con moderación. Por lo menos una vez por semana coma pescado.

\section{Cocinar con poca grasa}

Utilice sartenes con recubrimiento antiadherente, asi se puede evitar el uso de mucho aceite al freír. En la cocina los aceites grasos deben ser sustituidos, principalmente, por el aceite de colza (al freír) y el aceite de oliva (en las ensaladas).

\section{Bocadillos saludables}

La comida rápida (fastfood) y la ya elaborada son bombas de calorias. Renuncie a ellas. La naturaleza le ofrece ricos productos para las comidas entre horas como: uvas, zanahorias o manzanas.

\section{Bebidas saludables}

Evite las bebidas que contienen azúcar. Aplaque su sed con agua mineral, zumos de frutas o té de hierbas

\section{MÁS EJERCICIO EN SU VIDA COTIDIANA}

\section{Tómese tiempo}

Haga ejercicio diariamente por 30 o 60 minutos. Elija actividades que pueda acomodar en su vida cotidiana.

\section{Use el camino al trabajo como entrenamiento}

Use, por ejemplo, el tiempo de espera en la parada de autobús y tense los glúteos y luego los músculos del estómago, alternativamente. Después balancéese sobre los dedos del pie, subiendo y bajando. Tal vez le alcance el tiempo incluso para ir a pie hasta la próxima parada.

\section{Prefiera la bicicleta}

Para hacer gestiones en las cercanías use la bicicleta. Colóquela en un lugar a su alcance, de tal manera que la pueda usar en cualquier momento y manténgala apta para funcionar.

\section{Prueba con la dinámica de grupo}

Si le gusta hacer deporte en grupo, aprovéchelo. El establecer horas fijas para el deporte y el tener compañeros simpáticos puede ayudar a mantener la motivación en momentos de desánimo.

\section{Hacer ejercicio}

Escoja tipos de deporte con una intensidad leve hasta media de esfuerzo. El Nordic-Walking es un deporte ideal. Si usted suda levemente y puede conversar bien durante la práctica del deporte, entonces el esfuerzo que hace es el correcto.

\section{MANTÉNGASE ACTIVO PERMANENTEMENTE}

\section{Fijese objetivos realistas}

Objetivos que no son fáciles de lograr, nos hacen tener mala conciencia y nos desmotivan.

\section{Introducir días de acción}

De una a 3 veces por año debería crear condiciones claras, rompiendo costumbres antiguas y ordenando su casa radicalmente. Esto vale para el refrigerador, asi como para sótano y la sala. Cárguese de fuerza y energía para otros campos de la vida.

\section{Engáñese a sí mismo}

Solamente las medidas que son fáciles de cumplir pueden mantenerse en la vida cotidiana. Por ejemplo: las zapatillas de deporte que están en el corredor serán, probablemente, también usadas. Lo mismo sucede con el contenido del refrigerador: Los alimentos saludables colóquelos bien adelante ya que están más al alcance y son los primeros que se toman.

Fuente: Fundación alemana de Diabetes. Interpretación de datos Test de Findrisk.; 2017. Disponible en: https://www.diabetesstiftung.org/ gesundheitscheck-diabetes-findrisk 
ANEXO 5. Modelo de informe de comunicación con el médico y otros profesionales sanitarios

Farmacia (nombre, dirección y datos de contacto: teléfono/correo electrónico/web)

Fecha:

De: (nombre y apellidos y no colegiado del farmacéutico)

Para: Dr./a. (nombre y apellidos)

Estimado compañero, tras realizar:

A. El test de Findrisk y posteriormente una medida de hemoglobina glicosilada.

Se ha detectado que $D / D^{a}$. (nombre y apellidos del paciente y $n^{\circ} T S I$ )

presenta:

Un resultado en el test de Findrisk de y un valor de hemoglobina glicosilada de

He aconsejado al paciente que se ponga en contacto con usted para considerar la información anterior. Además, se propone

(si fuera necesario proponer algo). Por favor, ruego proporcione cualquier información relevante sobre el resultado.

Firma farmacéutico y $n^{\circ}$ colegiado: 
ANEXO 6. Hoja de recogida de datos básicos del paciente

Nombre y apellidos del paciente:

Teléfono y/o e-mail:

Sexo:

Fecha de nacimiento:

Datos antropométricos:

\begin{tabular}{|l|l|l|l|}
\hline & Visita 0 & Visita 4 & Visita 6 \\
\hline Peso $(\mathrm{kg})$ & & & \\
\hline Altura $(\mathrm{m})$ & & & \\
\hline $\mathrm{IMC}\left(\mathrm{kg} / \mathrm{m}^{2}\right)$ & & & \\
\hline
\end{tabular}

Alergias:

Hábitos nocivos (tabaco, alcohol):

Tipo de diabetes (tipo 1 o 2):

Años de evolución de diabetes:

Otros datos de interés: 


\begin{tabular}{|l|l|}
\hline \multicolumn{2}{|c|}{ Primera fase } \\
\hline V0 & \\
\hline V1 & \\
\hline V2 & \\
\hline V3 & \\
\hline V4 & \\
\hline Total & \\
\hline
\end{tabular}

Fase mantenimiento

\begin{tabular}{|l|l|}
\hline V5 & \\
\hline V6 & \\
\hline Total & \\
\hline
\end{tabular}

* Indicar tiempos en minutos. 
ANEXO 8. Escala de conocimientos en diabetes ECODI de Bueno y cols.

1. La insulina es:

a) Un medicamento que siempre recibimos del exterior y sirve para bajar el azúcar (o glucosa) en la sangre.

b) Una sustancia que eliminamos en la orina.

c) Una sustancia que segregamos en el organismo (en el páncreas) para regular el azúcar (o glucosa) en la sangre.

d) No sabe/no contesta.

2. Los niveles normales de azúcar [o glucosa) en sangre son:

a) $30-180$.

b) $70-140$.

c) $120-170$.

d) No sabe/no contesta.

3. Si usted nota aumento de sed, orinar con más frecuencia, pérdida de peso, azúcar en la orina, pérdida de apetito..., ¿qué cree que le ocurre?
a) Bajo nivel de azúcar (o glucosa) en sangre: hipoglucemia.
b) Niveles normales de azúcar (o glucosa) en sangre.
c) Alto nivel de azúcar (o glucosa) en sangre: hiperglucemia.
d) No sabe/no contesta.

4. Un alto nivel de azúcar (o glucosa] en sangre, también llamado hiperglucemia, puede producirse por

a) Demasiada insulina.

b) Demasiada comida.

c) Demasiado ejercicio.

d) No sabe/no contesta.

5. Si usted nota sudoración fría, temblores, hambre, debilidad, mareos, palpitaciones..., ¿qué cree que le ocurre?

a) Bajo nivel de azúcar (o glucosa) en sangre: hipoglucemia.

b) Niveles normales de azúcar (o glucosa) en sangre.

c) Alto nivel de azúcar (o glucosa) en sangre: hiperglucemia.

d) No sabe/no contesta.

6. Una reacción de hipoglucemia (bajo nivel de azúcar, o glucosa, en sangre), puede ser causada por:

a) Demasiada insulina o antidiabéticos orales (pastillas).

b) Poca insulina o antidiabéticos orales.

c) Poco ejercicio.

d) No sabe/no contesta.

7. ¿Cuántas comidas al día debe hacer un diabético?

a) Tres: desayuno, comida y cena.

b) Cinco: desayuno, almuerzo, comida, merienda y cena.

c) Comer siempre que tenga hambre.

d) No sabe/no contesta.

8. ¿Cuál de estos alimentos no debe comer nunca el diabético?

a) Carne y verduras.

b) Refrescos y bollerias.

c) Legumbres (p. ej.: lentejas) y pastas alimenticias (p. ej.: macarrones).

d) No sabe/no contesta.

9. ¿De cuál de estos alimentos puede comer, pero no debe abusar el diabético?

a) Carne y verduras.

b) Refrescos y bollería.

c) Legumbres y pastas alimenticias.

d) No sabe/no contesta.

10. Una dieta con alto contenido en fibra vegetal puede:
a) Ayudar a regular los niveles de azúcar en la sangre.
b) Elevar los niveles de colesterol en la sangre.
c) No satisfacer el apetito.
d) No sabe/no contesta.

11. Una buena fuente de fibra es:

a) Carne.

b) Verdura.

c) Yogurt.

d) No sabe/no contesta. 
12. Para conseguir un buen control de la diabetes, todos los diabéticos, en general, deben:
a) Tomar antidiabéticos orales. (pastillas).
b) Seguir bien la dieta.
c) Inyectarse insulina.
d) No sabe/no contesta.

13. ¿Por qué es tan importante que usted consiga mantenerse en su peso ideal (no tener kilos de más)?

a) Un peso adecuado facilita el control de la diabetes.

b) El peso apropiado favorece la estética ("la buena figura").

c) Porque podrá realizar mejor sus actividades diarias.

d) No sabe/no contesta.

14. La actividad corporal, largos paseos, la bicicleta o la gimnasia para usted:
a) Está prohibida.
b) Es beneficiosa.
c) No tiene importancia.
d) No sabe/no contesta.

\section{5. ¿Cuándo cree usted que debe hacer ejercicio?}
a) Los fines de semana o cuando tenga tiempo.
b) Todos los dias, de forma regular, tras una comida.
c) Sólo cuando se salte la dieta o coma más de lo debido.
d) No sabe/no contesta.

\section{Con el ejercicio físico:}

a) Sube el azúcar (o glucosa) en sangre.

b) Baja el azúcar (o glucosa) en sangre.

c) No modifica el azúcar (o glucosa) en sangre.

d) No sabe/no contesta.

17. El ejercicio es importante en la diabetes porque:
a) Fortalece la musculatura.
b) Ayuda a quemar calorías para mantener el peso ideal.
c) Disminuye el efecto de la insulina.
d) No sabe/no contesta.

18. Usted debe cuidarse especialmente sus pies, ya que:

a) Un largo tratamiento con insulina hace que se inflamen los huesos.

b) Los pies planos se dan con frecuencia en la diabetes.

c) Los diabéticos, con los años, pueden tener mala circulación en los pies (apareciendo lesiones sin darse cuenta).

d) No sabe/no contesta.

\section{Lo más importante en el control de la diabetes es:}
a) No olvidar el tratamiento, seguir bien la dieta y hacer ejercicio de forma regular.
b) Tener siempre azúcar en la orina para evitar hipoglucemias.
c) Tomar la misma cantidad de insulina o antidiabéticos orales (pastillas) todos los días.
d) No sabe/no contesta.

20. En la orina aparece azúcar (o glucosa) cuando:

a) El azúcar (o glucosa) sanguíneo es demasiado bajo.

b) El azúcar (o glucosa) sanguíneo es demasiado alto.

c) La dosis de insulina o pastillas es demasiado grande.

d) No sabe/no contesta.

21. ¿Por qué es tan importante saber analizarse la sangre después de obtenerla pinchándose un dedo?

a) Porque sabrá el azúcar (o glucosa) que tiene en sangre en ese momento.

b) Porque es más fácil que en la orina.

c) Porque sabrá si es normal y así podrá comer más ese día.

d) No sabe/no contesta. 
ANEXO 9. Cuestionario de satisfacción y retribución

1. ¿Volvería a utilizar este servicio?

- Seguro que sí

- Probablemente sí

- Puede que sí o puede que no

- Probablemente no*

- Seguro que no*

2. Si marcó una de las 2 últimas opciones*, por favor, díganos cuál o cuáles son sus razones por las que el servicio no le atrae

3. ¿Cree que este servicio debería ser remunerado al farmacéutico?

- $\mathrm{Si}^{*}$

- No

4. Si marcó que siं, ¿quién cree que debería remunerar este servicio?

- La Administración pública

- El paciente o usuario del servicio

- Ambos

5. Si marcó que sí ¿qué cantidad cree usted que debería percibir el profesional farmacéutico por este servicio?

- $10 €$

- $20 €$

- $30 €$

- Otra cantidad:

6. ¿Recomendaría este servicio a un amigo o familiar que lo precise?

- Sin duda lo recomendaría

- Lo recomendaría con reservas

- No lo recomendaría

7. En una escala de 1-5 (siendo 1 muy insatisfecho y 5 muy satisfecho), ¿cómo valoraría la utilidad de la aplicación móvil y el uso del glucómetro? Tachar valor elegido.

$\begin{array}{lllll}1 & 2 & 3 & 4 & 5\end{array}$


ANEXO 10. Control de las visitas a urgencias e ingresos hospitalarios

\begin{tabular}{|l|l|}
\hline V1 & Respuesta \\
\hline $\begin{array}{l}\text { ¿En los } 6 \text { meses anteriores a esta visita, ha acudido al } \\
\text { servicio de urgencias? }\end{array}$ & \\
\hline $\begin{array}{l}\text { En caso afirmativo, ¿se trató de urgencias en el Hospital } \\
\text { o en atención primaria?* }\end{array}$ & \\
\hline ¿Cuál fue el motivo? & \\
\hline ¿Cuáles fueron las pruebas realizadas? & \\
\hline $\begin{array}{l}\text { ¿Cuánto tiempo estuvo en el hospital o en el centro de } \\
\text { atención primaria? }\end{array}$ & \\
\hline
\end{tabular}

\begin{tabular}{|l|l|}
\hline $\mathbf{V} \mathbf{2 , V 3 , V 4 , V 5 , V 6}$ & Respuesta \\
\hline $\begin{array}{l}\text { ¿Desde la última visita, ha acudido al servicio de ur- } \\
\text { gencias? }\end{array}$ & \\
\hline $\begin{array}{l}\text { En caso afirmativo, ¿se trató de urgencias en el hospital } \\
\text { o en atención primaria?* }\end{array}$ & \\
\hline ¿Cuál fue el motivo? & \\
\hline ¿Cuáles fueron las pruebas realizadas? & \\
\hline $\begin{array}{l}\text { ¿Cuánto tiempo estuvo en el hospital o en el centro de } \\
\text { atención primaria? }\end{array}$ & \\
\hline
\end{tabular}

* En caso de que el paciente entre por atención primaria y se derive al servicio de urgencias del hospital, se considerará urgencias en el hospital. 
ANEXO 11. Calidad de vida

Marque con una cruz la respuesta de cada apartado que mejor describa su estado de salud en el día de HOY. No marque más de una casilla en cada grupo.

\section{Movilidad}

$1 \square$ No tengo problemas para caminar

$2 \square$ Tengo problemas leves para caminar

$3 \square$ Tengo problemas moderados para caminar

$4 \square$ Tengo problemas graves para caminar

$5 \square$ No puedo caminar

\section{Autocuidado}

$1 \square$ No tengo problemas para lavarme o vestirme

$2 \square$ Tengo problemas leves para lavarme o vestirme

$3 \square$ Tengo problemas moderados para lavarme o vestirme

$4 \square$ Tengo problemas graves para lavarme o vestirme

$5 \square$ No puedo lavarme o vestirme

3. Actividades cotidianas (Ejemplo: trabajar, estudiar, hacer las tareas domésticas, actividades familiares o actividades durante el tiempo libre)

$1 \square$ No tengo problemas para realizar mis actividades cotidianas

$2 \square$ Tengo problemas leves para realizar mis actividades cotidianas

$3 \square$ Tengo problemas moderados para realizar mis actividades cotidianas

$4 \square$ Tengo problemas graves para realizar mis actividades cotidianas

$5 \square$ No puedo realizar mis actividades cotidianas

\section{Dolor/Malestar}

$1 \square$ No tengo dolor ni malestar

$2 \square$ Tengo dolor o malestar leve

$3 \square$ Tengo dolor o malestar moderado

$4 \square$ Tengo dolor o malestar fuerte

$5 \square$ Tengo dolor o malestar extremo

\section{Ansiedad/Depresión}

$1 \square$ No estoy ansioso ni deprimido

$2 \square$ Estoy levemente ansioso o deprimido

$3 \square$ Estoy moderadamente ansioso o deprimido

$4 \square$ Estoy muy ansioso o deprimido

$5 \square$ Estoy extremadamente ansioso o deprimido

Las variables de calidad de vida relacionada con la salud se obtienen con un instrumento de medida propiedad del Grupo EuroQol: ๑ 2011 EuroQol Group. EQ-5D-5L $\mathrm{L}^{\mathrm{T}}$ is a trademark of the Euro0ol Group. 
- Nos gustaría conocer lo buena o mala que es su salud HOY.

- La escala está numerada del 0 al 100.

- 100 representa la mejor salud que usted se pueda imaginar.

- 0 representa la peor salud que usted se pueda imaginar.

- Marque con una X en la escala lo buena o mala que es su salud HOY.

- Ahora, en la casilla que encontrará a continuación escriba el número que ha marcado en la escala.

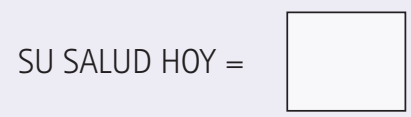




\section{ANEX0 12. Test Morisky-Green}

1. ¿Me olvido alguna vez de tomar la medicación? Si / No

2. ¿Tomo los medicamentos a las horas indicadas? Si / No

3. Cuando me encuentro bien, ¿dejo alguna vez de tomarlos? Si / No

4. Si alguna vez me siento mal, ¿dejo de tomar la medicación? Si / No

Para considerar un buen cumplimiento del tratamiento, la respuesta de todas las preguntas debe ser:

1. No, 2. Sí, 3. No, 4. No. 


\section{Servicio de revisión del uso \\ de los medicamentos (RUM)}

Fecha revisión ..................................... № RUM ...............

\section{DATOS DE LA FARMACIA}

Farmacia

Dirección

Teléfono.

e-mail ...

Nombre y apellidos

№ de colegiado

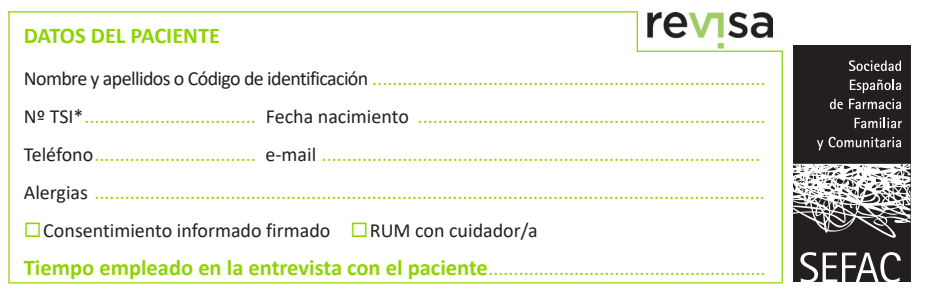

\begin{tabular}{|l|l|l|l|l|}
\hline PERCEPCIÓN & INDICACIÓN & EFECTOS & FALTA ADHERENCIA & POSOLOGÍA UTLIZZADA
\end{tabular}

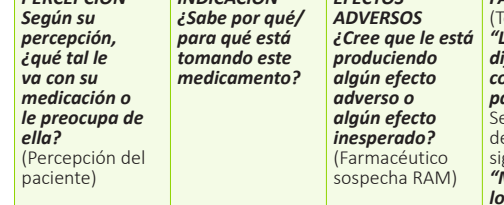

a mayoria de los pacientes tiene

dicultades para tomar TODOS sus

ara tomar los suyos?"

Seguir con la siguiente columna y en caso

(loiente pregunta:

los tratamientos speren dificitaltad en segur

cómo le va a usted?"

\begin{tabular}{l|l|l}
\hline INFORMACIÓn & $\begin{array}{l}\text { PRM (si procede) } \\
\text { ATENCIÓN }\end{array}$ \\
\hline
\end{tabular}

fita es la utilizada por el paciente y si el

- ausa, tiempo (¿¿adada cuánto? ? ¿Qué dias?)

- Administración lic Cómo lo toma? Incluye fraccionamiento,

- Dificultad de uso/toma (étiene alquún problema acerca de)

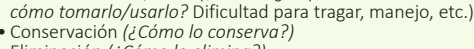

¿Hay algo más Rellenar solo en caso de detectar algún PRM no descrito

que le gustara de en los apartados anterores.

su medicación? RUM, registrar solo en caso de que afloren durante la

Indicar si el

información

adicional o algo
no le ha quedado
claro (dudas)

MEDICAMENTO 1 Nombre/CN**

\begin{tabular}{l|l|l}
\begin{tabular}{ll|l}
$\square$ Le va bien \\
$\square$ No le va bien
\end{tabular} & $\square$ Si & $\square$ No \\
& $\square$ No & $\square S i$ \\
& & Detallar: \\
& &
\end{tabular}

$\checkmark$ Si, falta adherencia parcia

Si, falta adherencia total

Posología prescrita .................................. Desde

\begin{tabular}{|c|c|c|c|c|c|}
\hline prescrita.. & & & & Indicado por & $(\mathrm{M}),(\mathrm{E}),(\mathrm{F}),(\mathrm{A}) * * *$ \\
\hline 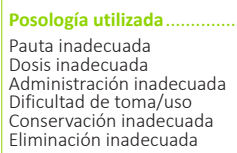 & 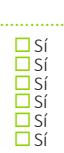 & $\begin{array}{l}\square \text { No } \\
\square \text { No } \\
\square \text { No } \\
\square \text { No } \\
\square \text { No } \\
\square \text { No }\end{array}$ & \begin{tabular}{|l|}
$\square$ No \\
$\square$ Si \\
Detallar:
\end{tabular} & $\begin{array}{l}\text { Q Contraindicación } \\
\text { Duplicidad } \\
\text { Errores en la dispensación } \\
\text { Errores en la prescripción } \\
\text { Dinteracciones } \\
\text { Medicamento no necesario } \\
\square \text { Otros PS que afectan al }\end{array}$ & $\begin{array}{l}\square \text { PS insuficientemente } \\
\text { tratado } \\
\square \text { Desabastecimiento } \\
\text { temporal } \\
\square \text { Falta de conciliación } \\
\square \text { Precaución de uso } \\
\square \text { Otro(s) }\end{array}$ \\
\hline
\end{tabular}

Notas complementarias

MEDICAMENTO 2 Nombre/CN**

\begin{tabular}{|l|l|l|}
\hline$\square$ Le va bien & $\square S i$ & $\square$ No \\
$\square$ No le va bien & $\square$ No & $\square S i$ \\
& & Detallar: \\
& &
\end{tabular}

Si, falta adherencia parcial

Sí, falta adherencia total

Causa de falta de adherencia:

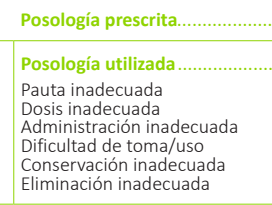

\begin{tabular}{|c|c|c|}
\hline 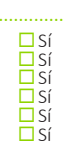 & $\begin{array}{l}\text { No } \\
\text { No } \\
\text { No } \\
\text { No } \\
\text { No } \\
\text { No } \\
\text { No }\end{array}$ & $\begin{array}{l}\square \text { No } \\
\square \text { Si } \\
\text { Detallar: }\end{array}$ \\
\hline
\end{tabular}

Indicado por (M), (E), (F), (A)****

MEDICAMENTO 3 Nombre/CN**

\begin{tabular}{l|l|l|l}
$\square$ Le va bien & $\square$ Si & $\square$ No & $\square$ Si, falta adherencia parcial \\
$\square$ No le va bien & $\square$ No & $\square S i$ & $\square S$ fi, falta adherencia total
\end{tabular} ai

Causa de falta de adherencia:

\begin{tabular}{l} 
Posología prescrita............. \\
\hline Posología utilizada........... \\
Pauta inadecuada \\
Dosis inadeccuada \\
Administración inadecuada \\
Dificultad dé toma/uso \\
Conservación inadecuada \\
Eliminación inadecuada
\end{tabular}

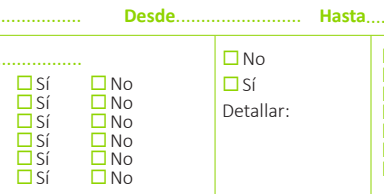

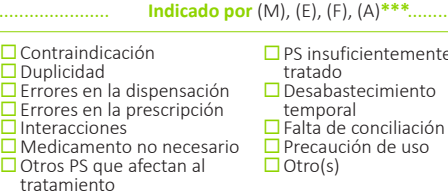

Notas complementaria

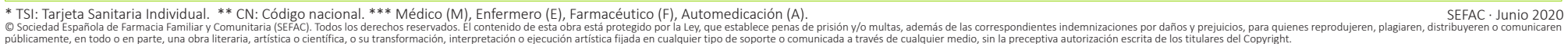




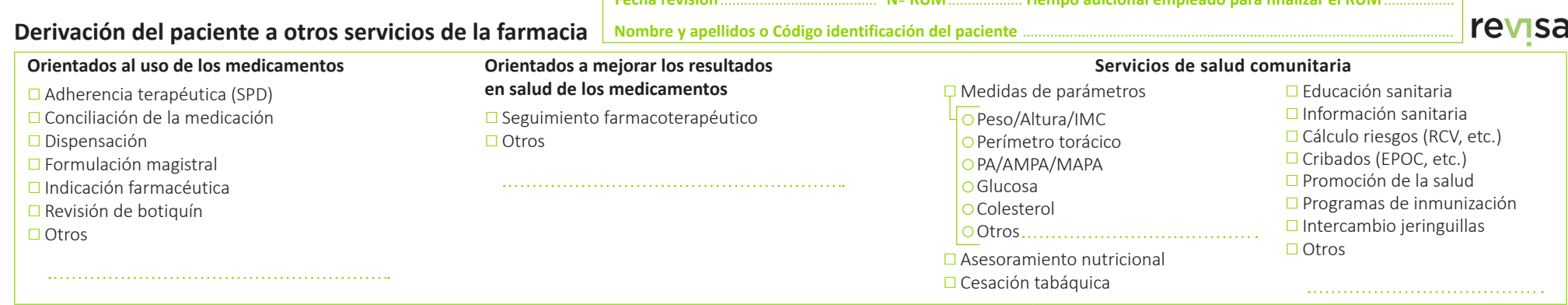

\section{Intervención general sobre el paciente}

$\square$ Facilitar información básica sobre educación sanitaria y estilos de vida saludables: alimentación, ejercicio físico, vacunación, revisiones de salud, evitar: tabaco, alcohol...

$\square$ Provisión de materiales de ayuda al paciente que ayuden al paciente a comprender y autogestionar su medicación y enfermedad

\section{Intervenciones sobre los medicamentos revisados}
MEDICAMENTO
Comentarios internos

- Facilitar IPM* y/o sugerencias de mejora en el proceso de uso del medicamento. Detallar:

$\checkmark$ Solucionar falta de adherencia. Detallar:

$\square$ Derivación a otro profesional sanitario**: $\square \mathrm{M} \quad \square \mathrm{E} \quad \square \mathrm{O}$. Detallar:

$\square$ Notificar tarjeta amarilla al SEFV-H. Detallar:

\section{MEDICAMENTO 2}

$\square$ Facilitar IPM* y/o sugerencias de mejora en el proceso de uso del medicamento. Detallar:

Solucionar falta de adherencia. Detallar:

$\square$ Derivación a otro profesional sanitario**: $\square \mathrm{M} \square \mathrm{E} \square \mathrm{O}$. Detallar:

$\square$ Notificar tarjeta amarilla al SEFV-H. Detallar: . .

$\neg$ Otros

\section{MEDICAMENTO 3}

- Facilitar IPM* y/o sugerencias de mejora en el proceso de uso del medicamento. Detallar:

$\checkmark$ Solucionar falta de adherencia. Detallar:

$\square$ Derivación a otro profesional sanitario**: $\square \mathrm{M} \quad \square \mathrm{E} \quad \square$ O. Detallar:

Notificar tarjeta amarilla al SEFV-H. Detallar:

$\square$ Otros

* Información personalizada del medicamento (IPM). ** Médico (M), Enfermero (E), Otro (O). dentistas, podólogos, optometristas, nutricionistas, etc. 


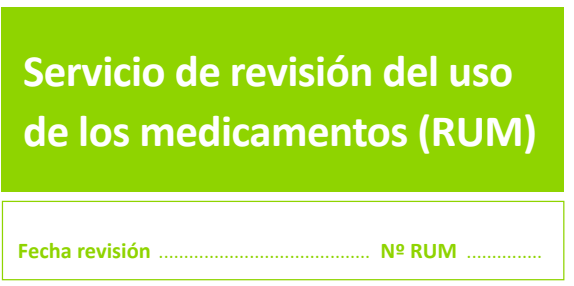

DATOS DE LA FARMACIA

revisa

Farmacia

Dirección

Teléfono

DATOS DEL PACIENTE

Nombre y apellidos

\section{INFORME PARA EL PACIENTE}

Información al paciente sobre la revisión de los medicamentos

Se han revisado los siguientes medicamentos:

Tras realizar una Revisión del Uso de los Medicamentos, le detallamos a continuación el informe de los medicamentos revisados.

\section{Acciones realizadas}

$\square$ Verificación de los medicamentos prescritos

$\neg$ Revisión de medicamentos indicados, de autocuidado y otros productos para su salud.

$\square$ Comprobación del grado de conocimiento de los medicamentos y su indicación.

$\square$ Comprobación de la pauta posológica (dosis, pauta y duración) respecto a la pauta prescrita.

$\neg$ Comprobación del conocimiento sobre la administración

$\neg$ Comprobación del conocimiento sobre la conservación y eliminación.

$\neg$ Provisión de la información personalizada sobre el proceso de uso de cada uno de dichos medicamentos.

Detección de Problemas Relacionados con los medicamentos y/o de Resultados Negativos asociados a los medicamentos.

$\checkmark$ Comprobación de la adherencia terapéutica según test de Haynes-Sackett.

$\square$ Provisión de materiales de ayuda al paciente que ayuden al paciente a comprender y autogestionar su medicación y enfermedad.

$\square$ Provisión de recomendaciones para un estilo de vida saludable.

$\checkmark$ Derivación al médico y/o a otros profesionales sanitarios. Indicar

$\square$ Derivación a otros servicios profesionales farmacéuticos asistenciales. Indicar

№ colegiado ............................... Fecha del informe 
ANEXO 14. Cuestionario de utilidad para el farmacéutico del glucómetro y aplicación móvil respecto al control glucémico y su educación sanitaria individualizada

1. En una escala de 1-5 (siendo 1 muy insatisfecho y 5 muy satisfecho), ¿cómo valoraría la utilidad de la aplicación móvil y el uso del glucómetro en función del tiempo en el adiestramiento? Tachar valor elegido.

$\begin{array}{lllll}1 & 2 & 3 & 4 & 5\end{array}$

2. En una escala de 1-5 (siendo 1 muy insatisfecho y 5 muy satisfecho), ¿cómo valoraría la utilidad de la aplicación móvil y el uso del glucómetro respecto a la educación sanitaria individualizada? Tachar valor elegido. 